\section{DIVISION E \\ COMMISSION 10}

\author{
PRESIDENT \\ VICE-PRESIDENT \\ PAST PRESIDENT \\ ORGANIZING COMMITTEE
}

\section{SOLAR ACTIVITY \\ ACTIVITÉ SOLAIRE}

\author{
Carolus J. Schrijver \\ Lyndsay Fletcher \\ Lidia van Driel-Gesztelyi \\ Ayumi Asai, Paul S. Cally, \\ Paul Charbonneau, Sarah E. Gibson, \\ Daniel Gomez, Siraj S. Hasan, \\ Astrid M. Veronig, Yihua Yan
}

\begin{abstract}
After more than half a century of community support related to the science of "solar activity", IAU's Commission 10 was formally discontinued in 2015, to be succeeded by C.E2 with the same area of responsibility. On this occasion, we look back at the growth of the scientific disciplines involved around the world over almost a full century. Solar activity and fields of research looking into the related physics of the heliosphere continue to be vibrant and growing, with currently over 2,000 refereed publications appearing per year from over 4,000 unique authors, publishing in dozens of distinct journals and meeting in dozens of workshops and conferences each year. The size of the rapidly growing community and of the observational and computational data volumes, along with the multitude of connections into other branches of astrophysics, pose significant challenges; aspects of these challenges are beginning to be addressed through, among others, the development of new systems of literature reviews, machine-searchable archives for data and publications, and virtual observatories. As customary in these reports, we highlight some of the research topics that have seen particular interest over the most recent triennium, specifically active-region magnetic fields, coronal thermal structure, coronal seismology, flares and eruptions, and the variability of solar activity on long time scales. We close with a collection of developments, discoveries, and surprises that illustrate the range and dynamics of the discipline.
\end{abstract}

\title{
1. Historical context
}

The IAU was founded in 1919, with the first General Assembly occurring in 1922 in Rome, Italy. The standing commissions that focused on what we nowadays capture under the term "solar physics" evolved over the following decades. A "Commission 10" was instituted by 1922, but under the title of "Solar Radiation". By 1935 that had been changed to "Sunspots and sunspot numbers", and by 1952 it had transitioned to "Photospheric phenomena". In parallel to Commission 10 there were the other solaroriented Commissions 11 through 15 dealing with subjects such as the solar atmosphere, eclipses, rotation, and spectroscopy (with, as noted in the 1961 report by Commission 10, a "lack of clear demarcation lines among the [three] solar commissions"). It was not until 1961 that "Solar Activity" became the title of Commission 10, lasting until its transition into Commission C.E2 in the overall reorganization of the IAU's structure in 2015. 
Table 1. Overview of Commission 10 leadership and triennial reports (as available in ADS) from 1961 onward, i.e. for the period that C10 operated under the banner of "Solar Activity" or "Activité Solaire". Reports flagged with an asterisk appear not to be available on line.

\begin{tabular}{|c|c|c|}
\hline Years & President and Vice President & ADS bibcode \\
\hline 1961 & Severny, A. B., Ellison, M. A. & $*$ \\
\hline $1964-1967$ & Svestka, Z., Jefferies, J. T. & $*$ \\
\hline 1967-1970 & Svestka, Z., Jefferies, J. T. & 1970IAUTA..14...71S \\
\hline 1970-1973 & Jefferies, J. T., Kiepenheuer, K. O. & 1973IAUTA..15 ...75J \\
\hline $1973-1976$ & Kiepenheuer, K. O., Newkirk, G. A. & \\
\hline 1976-1979 & Newkirk, G., Bumba, V. & 1979IAUTA..17b..11N \\
\hline 1979-1982 & Bumba, V., Tandberg-Hanssen, E. & 1982IAUTA \\
\hline $1982-1985$ & Tandberg-Hanssen, E., Pick, M. & 1985IAUTA..19...57T \\
\hline $1985-1988$ & Pick, M., Priest, E. R. & 1988IAUTA..20 ...55P \\
\hline 1988-1991 & Priest, E. R., Gaizauskas, V. & 1991IAUTA..21...53P \\
\hline 994 & skas, V., Engvold, O. & A..22...53G \\
\hline 1994-1997 & Engvold, O., Ai, G. & *1997IAUTA..23..121E \\
\hline $1997-2000$ & Ai, G., Benz, A. O. & * \\
\hline $2000-2003$ & Benz, A. O., Melrose, D. B. & * \\
\hline 2003-2006 & Melrose, D. B., Klimchuk, J. A. & 2007IAUTA..26...75M \\
\hline 2006-2009 & Klimchuk, J. A., van Driel-Gesztelyi, L. & 2009IAUTA..27...79K \\
\hline 2009-2012 & van Driel-Gesztelyi, L., Schrijver, C. J. & 2012IAUTA..28...69V \\
\hline $2012-2015$ & Schrijver, C. J., Fletcher, L. & (this report) \\
\hline
\end{tabular}

The scientific discipline focusing on solar activity continued to grow after Commission 10 settled on its final name, seeing among the many activities of its community the launch of a dedicated journal "Solar Physics" in 1967 and a marked advance in access to solar corona and inner heliosphere with the space-based Apollo Telescope Mount on Skylab in 1973. At present, we have ground- and space-based observatories looking at the Sun and innermost heliosphere from different perspectives, in a range of wavelengths, and probing the Sun's internal dynamics using helioseismology. But although these observatories provide a wealth of information and insight into the workings of our neighboring star, we struggle to provide the research community with a comprehensive view of the phenomena captured under the term "Solar Activity". That is certainly not a new challenge: for example, in the 1970-1973 report, then President Jefferies of C10 notes that "We believe that the major direction in which priority should be placed to facilitate the understanding of solar activity lies in the provision of space and ground based observatories specifically designed to complement each other's capabilities."

The early reports of Commission 10 published in the "Reports on astronomy" could highlight many of the key developments in the field overall. With the rapid growth of the discipline from the 1940s through the 1970s that quickly became impossible. From the middle of the 20th Century onward, severe selections had to be made in order for the task of the writing of a progress report to remain feasible. The need for such major downselects on what to cover clearly concerned the members of the Organizing Committees of the Commission (the presidents and vice-presidents are listed in Table 1). For example, in the report on the period 1967-1970, the Commission President Zdeñek Svestka apologizes for the "fairly severe" selection made in the Commission's overview with 32 pages of text as "only about one third of all published papers could have been mentioned in the references". By 2015, with some 6,000 papers appearing per triennium (as discussed in the next section), simply reading all published papers is too challenging a task. Even with severe selections applied by the Organizing Committee of Commission 10, the 19671970 report was still deemed too long: John Jefferies notes in the subsequent report for 
1970-1972 that the General Secretary requested that the reports should "concentrate on the more important developments" (which led to 34 pages of text in that cycle). The most recent reports condense the increased number of publications into 10 to 20 pages of text, selecting highlights only in the view of the members of the Organizing Committee.

For this report on 2012-2015 we would face a similarly daunting selection task. Instead, we chose to focus on three aspects of our community: the health of the community itself, new developments in the areas of instrumentation and IT infrastructure, and a discussion of scientific developments based on citations within the community and impressions of the OC members of C10.

\section{Trends in the research community and its publications}

As C10 transitions into C.E2 we take the opportunity to review the community's size and publication activity. For this, we use the tools provided by the Astrophysics Data System (ADS $\dagger$ ), which enables searches over all the major trade publications in astrophysics in general. We reviewed the number of refereed publications per year going back over a century, and quantified the population of active researchers and their publication productivity.

The study of phenomena related to "solar activity" often involves other aspects of the Sun (such as internal dynamics, dynamo, or surface field patterns) and they are obviously not limited to the Sun but drive phenomena throughout the heliosphere. We therefore do not attempt a separation by research disciplines along the somewhat arbitrary dividing lines between the IAU Commissions in what was Division II and is now Division E $\ddagger$, all the more so because of the shifts in focus of the Commissions related to solar physics since their inception after 1919 as noted above. Consequently, we searched ADS for abstracts of refereed publications in the "Astronomy" database, either mentioning the Sun or heliosphere or their synonyms. We filter out at least many of the papers that do not deal with Sun/heliosphere that come into the search results because their abstracts include, for example, a unit like "solar mass"; to do so, we exclude abstracts that contain one or more of the following words or word groups: cluster, dwarf, extrasolar, galaxy, gravitational, ice, kpc, solar system, stellar, binary, sunset, sunrise, eclipse, solar cell, solar occultation, interstellar medium, and supernova. Sampling the returned titles and abstracts suggests that the sample we study is dominated by far by papers that do indeed focus on Sun and heliosphere, while of course also including topics such as climate forcing, the physics of the upper atmospheres of planets, comets in the solar wind, cosmic-ray modulation, and weathering of lunar surface materials.

The ADS searches suggest that the productivity of the world-wide community researching the Sun and heliosphere continues to grow steadily if measured through its publications (Fig. 1). A rapid growth in the number of refereed publications that started after the Second World War continued up to about 1975. After that, the growth slowed drastically, transitioning to a sustained increase that doubles the number of refereed publications on a time scale of approximately 40 years, reaching a total of some 2200 refereed publications by 2014 .

Such automated searches enable us to process a lot of information, but it is not readily possible to avoid a distortion of the statistics associated with the author names. For one thing, authors with identical family names and initials for their given names are not differentiated. Also, authors who publish with different spellings or composites of

$\dagger$ URL: http://adsabs.harvard.edu/abstract_service.html

$\ddagger$ See http://www.iau.org/science/scientific_bodies/divisions/ 


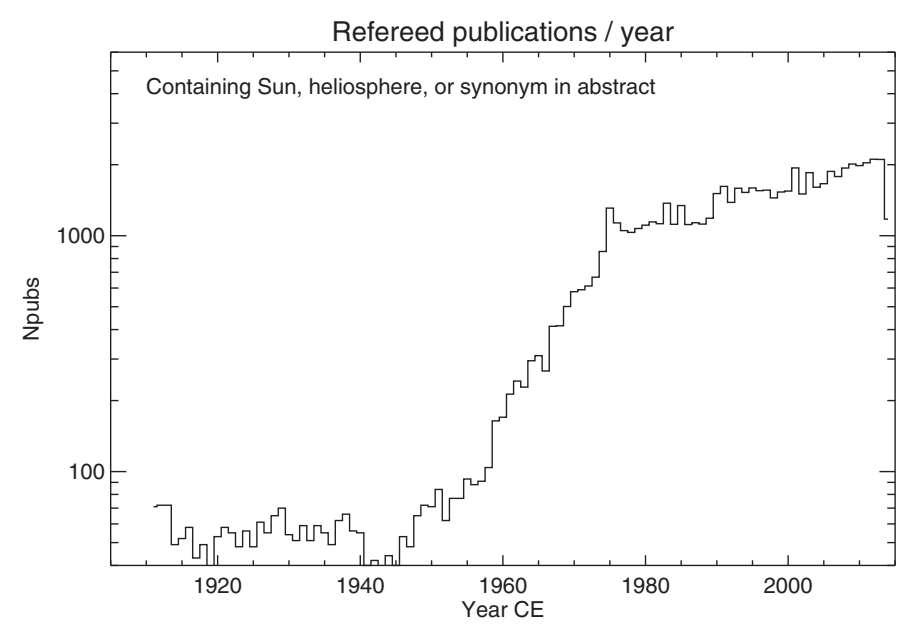

Figure 1. Number of refereed publications per year with abstracts focusing on Sun or heliosphere (as returned by ADS) from 1911 through 2014.

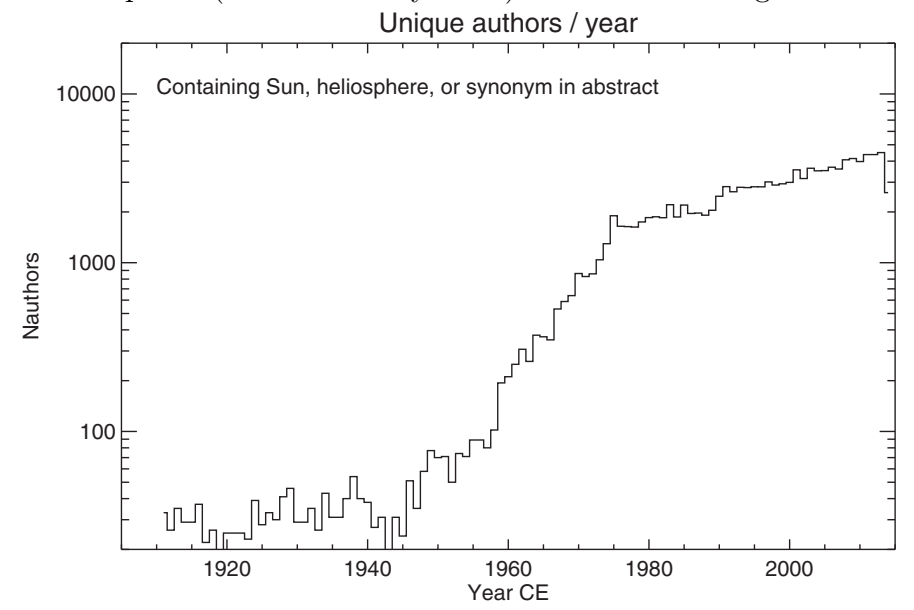

Figure 2. Number of unique authors for each year in publications per year with abstracts focusing on Sun or heliosphere (as returned by ADS) from 1911 through 2014.

their family names (e.g. married and maiden names) or their initials will be counted as separate individuals. These effects bias the total number of publications and researchers involved, but we expect their impacts to be limited when we review fractional trends over the years, as we do not expect very substantial changes of the relative impacts of these biases over the years.

The number of unique author names (subject to the above-noted caveat of the way we used the ADS system) contributing to refereed publications shows a trend that roughly mimics that of the number of publications: flat in the first half of the 20th Century with a total population of merely some 40 active researchers globally, then rapidly growing after WW II, and eventually growing exponentially from about 1975 onward up to a total approaching 5,000 . This growth rate of $\approx 2.5 \% / \mathrm{yr}$ is about twice the growth rate of the world's overall population (which averaged at $\approx 1.3 \% / \mathrm{yr}$ over the same period; Population reference bureau 2013), suggestive of a rather healthy growth in the research discipline of solar and heliospheric sciences and related fields over that of the general population. 


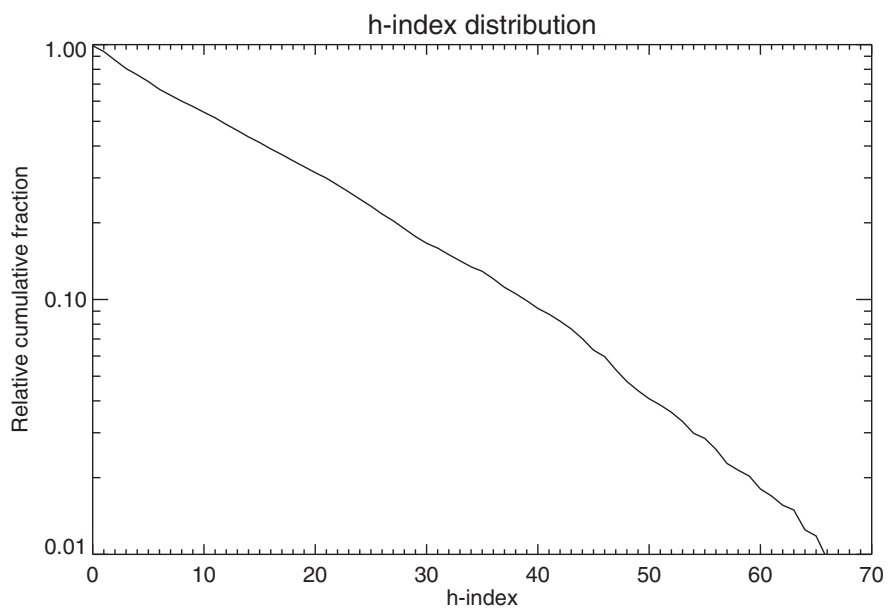

Figure 3. Distribution of $H$ indices for all authors publishing on Sun and heliosphere in 2014, based on ADS citation counts.

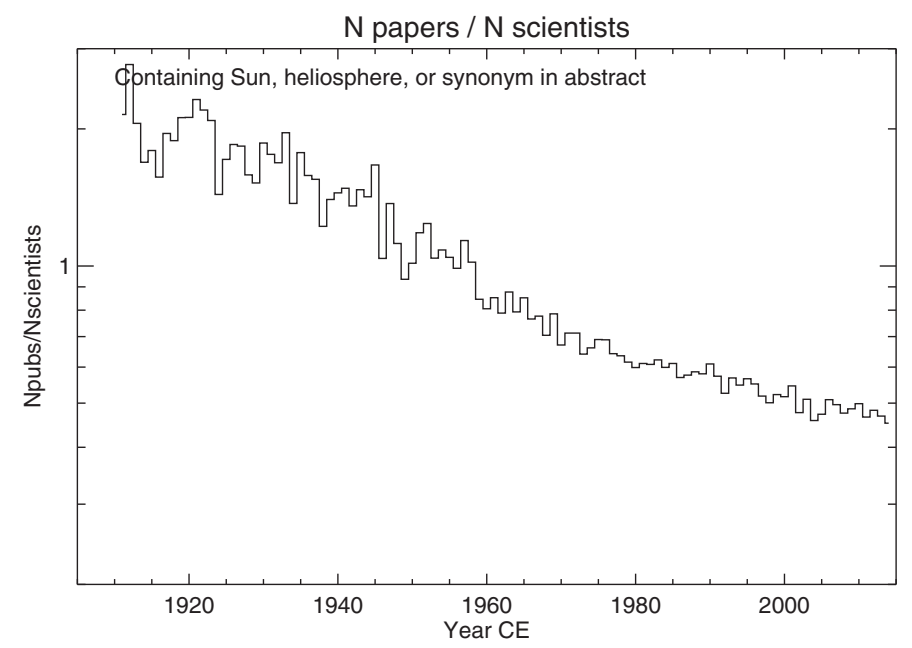

Figure 4. Number of refereed publications per author with abstracts focusing on Sun or heliosphere (as returned by ADS) from 1911 through 2014.

Many of the authors in the population publishing in the refereed literature are in the field only for a short time, or publish only infrequently as team members on, for example, instrument and facility papers. If we look for the population of authors who have worked in the field of solar-heliospheric sciences long enough to have contributed several papers that have been cited a few times, we can use the so-called $H$ index. That index is computed by ranking all refereed publications by an author in decreasing order of the number of citations, and then looking for the rank in that list where rank and number of citations equal. The downward-cumulative $H$ index distribution for authors publishing on Sun and heliosphere in 2014 (based on citations in ADS, and using refereed as well as unrefereed publications) is shown in Fig. 3.

For the present purpose of selecting researchers with a few years of activity in the field, we take a rather arbitrary $H$-index threshold of 5 , i.e., looking for authors with at least 5 refereed publications on their record that have each been cited at least 5 times. Fig. 3 shows that this includes about two thirds of all publishing authors in 2014. 


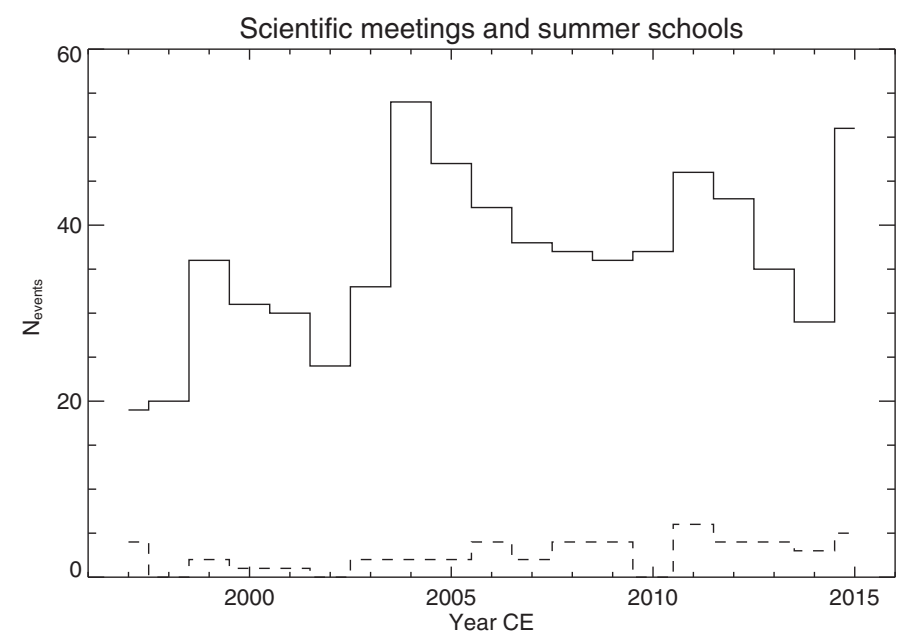

Figure 5. Number international scientific meetings (solid) and summer schools (dashed) from 1997 through 2014 (from the SOHO web site: http://sohowww.nascom.nasa.gov/community/).

Table 2. IAU meetings related to solar activity in the triennium 2012-2014.

\begin{tabular}{l}
2014 IAUS305 Polarimetry: the Sun to stars and stellar environments \\
2013 IAUS302 Magnetic fields throughout stellar evolution \\
2013 IAUS300 Nature of prominences and their role in space weather \\
2012 IAUS294 Solar and astrophysical dynamos and magnetic activity \\
\hline
\end{tabular}

The number of publications per year grows somewhat slower than the population of contributing authors, so that the number of publications per author shows a steady decrease over the past century, trending to just under half a refereed publication per year per author (see Fig. 4). That average value is subject to a large range for the population: some authors may not publish for years in a row, while others may exceed a dozen in particularly productive years, for example as members of sizable teams working with new instrumentation.

The community exchanges information efficiently at scientific meetings. Fig. 5 shows that the number of such events tends to increase over the years, with marked fluctuations from year to year, averaging around 40 meetings per year over the past decade. The Symposia supported by the IAU through Commission 10 in the last three years are listed in Table 2. Summer schools in which new generations of researchers are given broader or deeper views into the community's activities appear to grow slowly in frequency, trending towards about five events per year (dashed line in Fig. 5).

Although the scientific community working on "solar activity" appears healthy and growing, there is a clear need to improve how we communicate the excitement about our science to the general public: for example, only 8 in the most recent 400 press releases and news articles listed by the AAS $\dagger$ were related to some aspect of solar activity.

\section{Trends in observational capabilities}

The observational infrastructure enabling the study of solar physics has seen dramatic advances over the past five years. Among these, we highlight a few:

$\dagger$ http://aas.org/astronomy-in-the-news, accessed on 2015/09/03. 
In 2009, the two STEREO spacecraft (launched into Earth-trailing and Earth-leading orbits in 2006; Kaiser et al. 2008) passed the quadrature points relative to the Sun-Earth line. When combined with Earth-perspective viewing, the following years enabled a view of the entire solar surface, for the first time in history showing us the evolution of an entire stellar atmosphere.

The Atmospheric Imaging Assembly (AIA; Lemen et al. 2012) on board the Solar Dynamics Observatory (SDO - launched in 2010; Pesnell, Thompson, and Chamberlin 2012) provides uninterrupted observing of the outer atmosphere of the entire Earth-facing side of the Sun at a cadence of $12 \mathrm{~s}$ and a resolution of close to an arcsecond. Combined with magnetography and helioseismology with the Helioseismic and Magnetic Imager (HMI; Scherrer et al. 2012), as well as Sun-as-a-star spectroscopy in the EUV with the EUV Variability Experiment (EVE; Woods et al. 2012), this powerful SDO spacecraft sends down well over $1 \mathrm{~TB} /$ day. The primary data and higher-level derivatives fill a data archive that now exceeds $7 \mathrm{~PB}$ and holds over $96 \%$ of all data ever taken from space in the domain that focuses on the Sun and the physics of the Sun-Earth connections.

Instrumentation with high spatial or spectral resolution is flown on the JAXA-led Hinode/Solar-B mission (launched in 2006; Kosugi et al. 2007) and the NASA Small Explorer IRIS (Interface Region Imaging Spectrograph, launched in 2013; De Pontieu et al. 2014b) that offer images with resolutions between 0.1 and 0.3 arcsec, combined with spectroscopy in the visible and ultraviolet. RHESSI (Lin et al. 2002), launched in 2002 , is continuing to provide unique spectroscopic images of the Sun at high energy.

These space-based instruments provide important access to the domain from photosphere to corona, critically complemented by ground-based telescopes and their instrumentation, as well as by rocket experiments such as Hi-C (Winebarger et al. 2014).

In the optical domain, the $1.6 \mathrm{~m}$ New Solar Telescope (NST, Goode and Cao 2012) at Big Bear in the U.S.A. and the 1.5-meter German GREGOR solar telescope at Tenerife in Spain (Schmidt et al. 2012) have been in regular operation since 2012. The 1-m New Vacuum Solar Telescope (NVST, Liu et al. 2014c) at Fuxian Lake in southwest China is also in regular operation recently. CRISP (Crisp Imaging Spectro-polarimeter) at the Swedish 1-m Solar Telescope (SST; van Noort and Rouppe van der Voort 2008) reached 0.13 arcsec spatial resolution and high polarimetric sensitivity aided by post-processing. All these telescopes have capacities close to their diffraction limit due to advanced designs and excellent seeing conditions. We have seen glimpses into very high-resolution groundbased coronal observing as well with, for the first time, synoptic observations of coronal Stokes polarimetry in the near infrared by the Coronal Multichannel Polarimeter (CoMP, Tomczyk et al. 2008) telescope.

In the radio domain, the recently commissioned Chinese Spectral Radioheliograph (CSRH; Yan et al. 2009; Wang et al. 2013) at Mingantu in Inner Mongolia of China (renamed as Mingantu Ultrawide Spectral Radioheliograph-MUSER) is a radioheliograph operating with the widest frequency range ever reached from $400 \mathrm{MHz}$ to $15 \mathrm{GHz}$, with a high temporal, spatial, and spectral resolution. Recent non-solar dedicated radio arrays such as the Murchison Wide-field Array (MWA, Oberoi et al. 2011) and the LowFrequency Array for Radio astronomy (LOFAR, Mann, Vocks, and Breitling 2011) have obtained spectroscopic solar imaging at metric and lower frequencies. The recently upgraded Karl G. Jansky Very Large Array (EVLA) has provided solar radio dynamic imaging spectroscopy of type III bursts at decimeter wavelengths (Chen et al. 2013). Even the millimeter domain and beyond is seeing major advances with the Atacama Millimeter/sub-millimeter Array (ALMA; Bastian et al. 2015) observatory coming on line for solar observing as it continues to complete its construction phase. 
The observations of tens of thousands of Sun-like stars by the NASA Kepler satellite is offering yet more insights into the physics of the Sun, ranging from an improved understanding of internal structure and dynamics (with asteroseismic techniques) to a view of rare, extremely energetic flares. Kepler data, with ground-based follow-up studies, suggest that solar flares may occur with energies up to several hundred times higher than observed directly in the past half century with space-based instrumentation (Schrijver and Beer 2014).

\section{Trends in IT infrastructure for research and data}

The volume of information that needs to be processed by solar researchers is increasing rapidly. In terms of data to be analyzed, we have definitely reached the petabyte era. This is true for observational data in the archives of SDO, but also in computer experiments in which single snapshot data dumps of the advanced codes can exceed a TB.

A tendency towards open data policies means that we have ever more access to large volumes and a daunting diversity of data. That complicates finding, processing, and integrating data. Infrastructure support for, for example, the Virtual Solar Observatory (VSO), SolarSoft IDL (SSWIDL), and the Heliophysics Events Knowledgebase and Registry (HEK, HER) are critically important to enable efficient utilization of the growing data diversity and volume. The community lags in strategic thinking about these meta-infrastructural elements, both where current support and future expansion or replacement are concerned. There has also been a recent movement towards open-source data analysis software, with the development of the SunPy solar analysis environment in Python (Mumford et al. 2013).

A similar flood of information is found with scientific publications, which exceed 2,000 refereed publications per year (see above). Here, the support infrastructure of ADS is of critical value. The wide diversity of journals in which the works of colleagues are published requires subscription access to many publications, at costs that are increasingly hard to bear for relatively small research groups; here, preprint services such as arXiv and MaxMillennium play significant roles in making the community aware of what is going on. "Living reviews" as offered by the free on-line journal Living Reviews in Solar Physics enable new researchers to understand the context of their work and established researchers in one sub-specialty a quick introduction into adjacent areas by their peers.

Among the difficulties the solar activity community also faces is that many solar and inner-heliospheric events are studied by different groups and published in different journals. Finding studies on a particular solar region of interest is hampered by inconsistent use of the characterizing spatio-temporal coordinates of events which may be found in abstract, main text, tables, appendices, and sometimes only marked in figures that are not machine-readable. The IAU adopted a standard convention for this in 2009 (Leibacher et al. 2010) in its SOL standard (short for Solar Object Locator). Its use is encouraged by journal editors including those of Solar Physics, the Astrophysical Journal, and the Journal of Geophysical Research. Broad usage of the SOL standard would enable computer searches of related publications, enabling researchers to put new (meta-)studies in broader contexts.

\section{Trends in research directions and key findings}

For the highlights touched upon in this report, we opted for two criteria to identify topics of interest. One is to mention specific areas of note in the opinion of the Organizing Committee that may be new developments, are highly specialized yet significant, may 
concern new instrumentation or methods, or are otherwise deemed to be developments that may grow to see more activity in terms of publications in the near future.

The other criterion we applied is to be guided towards topics of frequent activity by the community itself by looking at the most cited works. Such a selection does introduce a bias toward the papers published early in the period reviewed, of course, but our purpose is not to identify the most-cited works per se, but rather to find the dominant themes within the set of these works that apparently resonate strongly within the community already within the $3-y$ window from which they are selected.

We searched for the most-cited refereed publications from ADS with the terms "solar.activity", "coronal.mass.ejection", "solar flare", "solar prominence", or synonym(s) in their abstracts in the period 2012-2014. Within this set, we identify the following themes (sorted alphabetically): active-region magnetic fields; coronal thermal structure; coronal seismology; solar flares and eruptions; and the Sun-in-time related aspects of long-term solar variability including cosmic-ray modulation. We close with a collection of developments, discoveries, and surprises.

\subsection{Active-region magnetic fields}

The photospheric magnetic field of active regions forms the foundation of the overlying atmosphere. Its evolution - through emergence, displacement, and submergence of flux is key to driving eruptive and explosive events in the corona and into the heliosphere. Until recently, generally only line-of-sight magnetic field maps were available for this work (with its routine observations enabling the study of increasingly large samples; one example of a large study is the work by Al-Ghraibah, Boucheron, and McAteer 2015, who analyse the magnetic properties of $\sim 2,000$ active regions looking for signatures likely involved in flaring). Nowadays, regular vector-magnetic determinations from the observed polarization signals are available from sources that include Hinode, SOLIS, and SDO/HMI. The sensitivity of such vector field maps allows the detection of lasting changes in the photospheric field when comparing pre- to post-flare observations (e.g. Wang et al. 2012). Temporal resolution is so good that coronal events can be tightly bracketed to try to understand the causes of flares and eruptions, as well as the changes in energy and helicity involved; for example, Sun et al. (2012) analyze a series of nonlinear force-free field models for the evolution in the energy of an active region around the time of a major eruption.

The increasing availability of vector-magnetic data enables statistical studies on the properties of active regions and their activity heretofore possible only on line-of-sight magnetograms. For example, Bobra and Couvidat (2015) use a data base of vector field maps of over 2,000 active regions to train a machine-learning algorithm to attempt forecasting of large flares. Su et al. (2014) review 3,000 vector field maps of some 60 regions to compare estimates of free energy with flare rates. Tiwari et al. (2015) analyze a sample of nearly 200 coronal mass ejections to reveal that flux, twist, and proxies for free energy tend to set upper limits to the speed of CMEs emanating from the active regions studied. Otsuji, Sakurai, and Kuzanyan (2015) review hundreds of vector magnetograms of a sample of 80 active regions to study helicity and twist parameters to test for the influence of Coriolis forcing.

One long sought-after goal of flare and CME physics is to use models of the solar atmospheric field to understand why field configurations destabilize and under what conditions destabilization begins and proceeds. In a "meta-analysis" review, Schmieder, Aulanier, and Vršnak (2015) discuss recent developments, including the use of 3D field extrapolations that suggest that topological structures (notably null points and hyperbolic flux 
tubes) may be involved in the triggering and generally as tracers of reconnection processes early on.

But even as the availability of vector-magnetic field maps becomes routine, the realization is growing that by themselves they appear insufficient to provide generally adequate lower-boundary conditions to understand the solar atmospheric activity. For example, a group of modelers using a variety of non-linear force-free field algorithms concludes in a series of studies (see, e.g. DeRosa et al. 2009; Tadesse, Wiegelmann, and MacNeice 2015; DeRosa et al. 2015, and references therein) that snapshot vector-magnetic maps do not suffice to obtain a reliable coronal field model with accurate energy or helicity measurements, with effects of instrumental resolution and field of view, as well as the model geometry (cartesian vs. spherical) all compounding the problems. New developments include the use of coronal loop observations to guide non-potential field models either from a single perspective as is possible currently (e.g. Malanushenko et al. 2014) or by using stereoscopic data from existing or future space- based instrumentation such as STEREO, Solar Orbiter, and SDO (e.g. DeRosa et al. 2009; Aschwanden, Schrijver, and Malanushenko 2015). Methods to follow the evolution of active-region fields based on data driving are also being developed, using the uninterrupted stream of (vector) magnetograms now available from space-based platforms (e.g. Cheung and DeRosa 2012; Fisher et al. 2015, based on the MHD-like magnetofrictional approximation), even reaching up to global MHD field models from near the solar surface into the heliosphere (Hayashi et al. 2015). Others are developing MHD methods to study CME initiation based on observed surface field evolution (e.g. Amari, Canou, and Aly 2014). Fundamental difficulties with these emerging methods include the difficulty in measuring the transverse field in areas of relatively low flux densities, the intrinsic 180-degree ambiguity in the field direction given a magnitude for the transverse component, the need to constrain the electric field to drive the model's evolution, and ultimately the quantitative comparison with solar observables to determine the verisimilitude of the model fields.

Even as our ability to observe and process rapidly growing data volumes on active region fields increases, we remain puzzled by the Sun's atmospheric magnetic field: we have yet to understand how large amounts of energy can sometimes be stored to eventually be explosively converted into flares and CMEs, while in other cases the energy is either not stored or is not explosively released. For a recent review of our understanding of the magnetic field in the solar atmosphere, and the variety of methods used to observe and study it, we refer to Wiegelmann, Thalmann, and Solanki (2014), and references therein.

\subsection{Coronal thermal structure and heating}

The X-ray solar corona is made of complex arrays of magnetic flux tubes anchored on both sides to the photosphere, confining a relatively dense and hot plasma. This optically thin plasma is almost fully ionized, with temperatures above $1 \mathrm{MK}$, and emitting mostly in the extreme UV to X-rays with intensities proportional to the square of its density. Significant progress was made in studying the physical and morphological features of coronal loops in a series of very successful EUV and X-ray solar missions, since the first observational evidence of the presence of coronal loops provided by rocket missions in the mid-1960s (Giacconi et al. 1965).

In recent years, several solar missions were launched and started producing spectacular images and data in different spectral ranges. The high spatial and temporal resolution of these instruments and the complementarity between these data sets, poses new challenges to understand the heating and dynamics of coronal loops. The launch in 2010 of the Solar Dynamics Observatory (SDO, Pesnell, Thompson, and Chamberlin 2012) allows continuous observation of the whole Sun with high temporal and spatial resolution with 
AIA, EVE, and HMI. In particular, AIA images span at least 1.3 solar diameters in multiple wavelengths, at about 1 arcsec in spatial resolution and at a cadence of about 10 seconds. Coronal loops observed by AIA have been found to be highly variable and highly structured in space, time, and temperature, challenging the traditional view of these loops as isothermal structures and favoring the case for multi-thermal cross-field temperature distributions. Because the thermal conductivity is severely reduced in the directions perpendicular to the magnetic field, a spatially intermittent heating mechanism might give rise to a multi-threaded structure in the internal structure of loops. One possible example of such an intermittent heating process is MHD turbulence, which is expected to produce fine scale structuring within loops all the way beyond the resolution of current observations (Gómez, Martens, and Golub 1993; van Ballegooijen, AsgariTarghi, and Berger 2014). A recent study by Brooks, Warren, and Ugarte-Urra (2012) combining spectroscopic data from the EUV Imaging Spectrometer (EIS, Culhane et al. 2007) aboard Hinode and SDO/AIA images, shows that most of their loops must be composed of a number of spatially unresolved threads.

More recently, the sounding rocket mission High-resolution Coronal Imager (Hi-C, Cirtain et al. 2013), achieved an unprecedented spatial resolution 0.2 arcsec in EUV images. Winebarger et al. (2014) find that the finely structured corona, down to the 0.2 " resolution, is concentrated in the moss and in areas of sheared field, where the heating is intense. This result suggests that heating is on smaller spatial scales than AIA and that it could be sporadic. These results are consistent with differential emission measure (DEM) analysis that study the distribution of temperature across loops. Warren, Winebarger, and Brooks (2012) present a systematic study of the differential emission measure distribution in active region cores, using data from EIS and the X-Ray Telescope (XRT) aboard Hinode. Their results suggest that while the hot active region emission might be close to equilibrium, warm active regions may be dominated by evolving million degree loops in the core. More recently, Schmelz et al. (2014) used XRT and EIS data as well as images from SDO/AIA, and found that cooler loops tend to have comparatively narrower DEM widths. While the DEM distribution of warm loops could be explained through bundles of threads with different temperatures, cooler loops are consistent with narrow DEMs and perhaps even isothermal plasma. The authors then speculate that warm, multi-thermal, multi-threaded loops might correspond to plasma being heated, while cool loops are composed of threads which have had time to cool to temperatures of about a million degrees, thus resembling a single isothermal loop.

The Interface Region Imaging Spectrograph (IRIS, De Pontieu et al. 2014) was launched in 2013, and provides crucial information to understand coronal heating, by tracing the flow of energy and plasma from the chromosphere and transition region up to the corona. IRIS obtains high resolution UV spectra and images with high spatial $(0.33$ arcsec $)$ and temporal (1 s) resolution. Recent IRIS observations show rather fast variations (20-60 s) of intensity and velocity on spatial scales smaller than $500 \mathrm{~km}$ at the foot points of hot coronal loops (Testa et al. 2014). These observations were interpreted as the result of heating by electron beams generated in small and impulsive heating events (the so-called coronal nanoflares).

Theoretical models of coronal heating have been traditionally classified into AC or DC, depending on the time scales involved in the driving at the loop foot points: (a) $\mathrm{AC}$ or wave models, for which the energy is provided by waves at the Suns photosphere, with timescales much faster than the time it takes an Alfvén wave to cross the loop; (b) DC or stress models, which assume that energy dissipation takes place by magnetic stresses driven by slow foot point motions (compared to the Alfvén wave crossing time) at the Suns photosphere. Although these scenarios seem mutually exclusive, two common 
factors prevail: (i) the ultimate energy source is the kinetic energy of the sub-photospheric velocity field, (ii) the existence of fine scale structure is essential to speed up the dissipation mechanisms invoked (Gómez 2010). For a coronal heating mechanism to be considered viable, the input energy must be compatible with observed energy losses in active regions, estimated by Withbroe and Noyes (1977) to be $\approx 1 \times 10^{7} \mathrm{erg} \mathrm{cm}^{-2} \mathrm{~s}^{-1}$. Welsch (2015) used high-resolution observations of plage magnetic fields made with the Solar Optical Telescope aboard Hinode to estimate the vertical Poynting flux being injected into the corona, obtaining values of about $\approx 5 \times 10^{7} \mathrm{erg} \mathrm{cm}^{-2} \mathrm{~s}^{-1}$, which suffices to heat the plasma.

\subsection{Coronal seismology}

Coronal seismology, like terrestrial seismology and traditional helioseismology, provides a means of probing the background state of the medium through which the waves propagate. Although the corona is not hidden from view like the interiors of Earth and Sun, it is very difficult to directly measure plasma and magnetic properties such as density $\rho$, magnetic field $\mathbf{B}$, or transport coefficients. Observations of oscillations in the coronal plasma can potentially provide powerful constraints on these quantities, for example through the Alfvén velocity B $/ \sqrt{\mu \rho}$ (e.g., see the Living Review of Nakariakov and Verwichte 2005).

But unlike the solar interior and terrestrial examples where waves are but perturbations on the background state, the coronal seismic waves are crucially important to the energy balance of their host medium. Coronal heating and solar wind acceleration are widely thought to result at least in part from waves (Ofman 2010). An overview of coronal seismology as of 2012 is provided by De Moortel and Nakariakov (2012).

The last 5-8 years have seen an explosion in coronal wave studies due to the advent of new instrumentation, such as the ground-based Coronal Multichannel Polarimeter (CoMP, Tomczyk et al. 2008) and the space-based Atmospheric Imaging Assembly (AIA) on the Solar Dynamics Observatory (SDO). Both have revealed ubiquitous Alfvén-like (i.e., transverse to the magnetic field) coronal oscillations (Tomczyk et al. 2007; McIntosh et al. 2011, respectively), though the interpretation of their exact natures - Alfvén or kink - is controversial (Van Doorsselaere, Nakariakov, and Verwichte 2008). The term "Alfvénic" is commonly used to encompass both wave types. In either case, the increased resolution of the AIA observations (White and Verwichte 2012) has allowed the detection of sufficient oscillatory power to potentially power both the corona and solar wind (McIntosh et al. 2011), though precise mechanisms are not currently known with certainty. The concentration of oscillatory power in the few-minute period band, and in particular at around 5-minutes, strongly suggests a link with the Sun's internal $p$-mode global oscillations. Consistency in the estimation of Alfvén speed between seismic techniques and magnetic field extrapolation is confirmed by Verwichte et al. (2013) using AIA data from a flare-induced coronal loop oscillation.

Time-Distance techniques applied to CoMP observations indicate a preponderance of outward propagating waves over inward propagation, even in closed loop structures, suggesting in situ dissipation (or mode conversion) on a timescale comparable to the Alfvén crossing time (Tomczyk and McIntosh 2009).

Disentangling Alfvén and kink waves is addressed in depth by Mathioudakis, Jess, and Erdélyi (2013). In the magnetically structured solar atmosphere, the only true Alfvén wave is torsional, and there is considerable interest in identifying these in observations because of the amount of energy they could potentially contribute to the outer atmosphere. However, being incompressive, Alfvén waves are not seen in intensity, and torsional Alfvén waves are also difficult to detect in Doppler (De Moortel and Pascoe 2012; McIntosh and De Pontieu 2012). Recently though, 0.33-arcsec high-resolution 
observations of the chromosphere and transition region (TR) with NASA's Interface Region Imaging Spectrograph (IRIS), coordinated with the Swedish Solar Telescope, have revealed widespread twisting motions across quiet Sun, coronal holes, and active regions alike that often seem to be associated with heating (De Pontieu et al. 2014a). This must presumably extend to the corona as well.

Dissipation of Alfvén waves in the solar atmosphere has long been thought to rely largely on the generation of Alfvén turbulence through nonlinear interaction between counter-propagating waves (Cranmer and van Ballegooijen 2005; van Ballegooijen et al. 2011). Observations with CoMP showing enhanced high-frequency power near the apex of coronal loops (Liu et al. 2014a; De Moortel et al. 2014) possibly supports this view.

The presumed acceleration of the solar wind by Alfvén turbulent energy deposition poses the challenge of identifying and explaining counter-propagating Alfvén waves in open magnetic field regions required to produce that turbulence. Morton, Tomczyk, and Pinto (2015) confirm the presence of these counter-propagating waves using CoMP. These observations also provide evidence of a link to the $p$-mode spectrum, which presumably relies on magnetoacoustic-to-Alfvén mode conversion occurring in the lower atmosphere (Cally and Hansen 2011; Hansen and Cally 2012).

\subsection{Flares}

\subsubsection{The flare's impact on the lower solar atmosphere}

The solar chromosphere is where most of the energy of a solar flare is dissipated and radiated in bright linear structures called flare ribbons, and recent work on this topic has been dominated by observations from IRIS. The high spectral resolution available with IRIS shows complex spectral line profiles in the Si IV transition region line at $1394 \AA$ and $1403 \AA$ sometimes with two or three time-varying gaussian components within one IRIS spatial pixel (Brannon, Longcope, and Qiu 2015). The hot "coronal" line of Fe XXI $(1354 \AA)$ on the other hand shows only a single strongly blue-shifted component (Brosius and Daw 2015; Graham and Cauzzi 2015) originating in compact ribbon sources at the beginning of a flare, with no hot "stationary component" present. The presence of this line also demonstrates the high temperatures reached by the chromosphere in flares as deduced from Hinode/EIS observations (Brosius 2013; Graham et al. 2013). With EIS, we see 1.5-3 MK redshifts (Young et al. 2013) confirming earlier reports, as well as significant non-thermal broadening. This means that the momentum-conserving condensation front that is produced by flare heating and paired with the evaporation flow contains hot plasma. In turn this implies that the condensation front originates relatively high in the chromosphere, otherwise such high temperatures would not be possible in the standard (electron-beam-driven) model of flares. This is somewhat at odds with recent measurements of element abundance (Warren 2014) which look more photospheric than coronal, suggesting that up-flowing evaporated material comes from low down in the chromosphere, below where the normal fractionation by first ionization potential sets in.

Flare optical (or white-light - WL) emission continues to be difficult to observe and difficult to explain. Optical foot points characterized using 3-filter observations with Hinode/SOT (Watanabe et al. 2013; Kerr and Fletcher 2014) could be explained by modest temperature increases of the photospheric black body. The other main proposed radiation mechanism is recombination emission, and flare continuum in the near UV (beyond the Balmer edge) observed with IRIS has an intensity consistent with this (Heinzel and Kleint 2014), but the tell-tale Balmer jump has not been seen. Co-spatial hard X-ray (HXR) and white-light flare sources have been observed using RHESSI and SDO/HMI, and require that both emissions are produced a few hundred kilometres above the photosphere (Martínez Oliveros et al. 2012; Krucker et al. 2015), at a height corresponding 
to the temperature minimum region, and beyond the range expected for HXR-emitting electrons arriving from the corona (unless the chromosphere is under-dense compared to expectations). It may be possible to generate optical emission from the temperature minimum region, for example by modest heating due to ion-neutral damping (Russell and Fletcher 2013), but the presence of non-thermal electrons in this plasma is harder to explain. Also indicating the flare's impact on the dense lower atmosphere, there have been many more reports of "sun quakes" — flare seismic emission (e.g. Alvarado-Gómez et al. 2012; Zharkov et al. 2013) — but the mechanical driver remains uncertain; there are correspondences with either HXR sources (pressure pulses from electron-beam driven shocks) or magnetic transients (Lorentz forces) in some but not all cases. High-resolution ground-based flare observations using the New Solar Telescope at BBSO show extraordinary fine structure, on a sub-arcsecond scale, in flare ribbons and footprints (Deng et al. 2013; Sharykin and Kosovichev 2014), setting the scene for future observations with DKIST.

\subsubsection{Magnetic-field evolution and energetics}

Examination of the changes in photospheric vector field occurring at the flare impulsive phase by Petrie $(2012,2013)$ using the HMI on SDO suggests strong, permanent and abrupt variations in the vertical component of the Lorentz force at the photosphere consistent with a downward 'collapse' of magnetic loops, and changes in the horizontal component mostly parallel to the neutral line in opposite directions on each side, indicating a decrease of shear near the neutral line. The brighter the flare (as expressed in the GOES class), the larger are both the total (area-integrated) change in the magnetic field and the change of Lorentz force (Wang, Liu, and Wang 2012), although Su et al. (2014) found that indicators of magnetic non-potentiality (e.g. rotation, shear and helicity changes) are more closely associated with flares - see for example the study of flare-associated rotating sunspots by Vemareddy, Ambastha, and Maurya (2012). In the corona, imaging and spectroscopic observations show compelling evidence for plasma flows suggestive of those expected around a coronal reconnection region (e.g. Tian et al. 2014; Su et al. 2013).

The growing number of observed flares in archives and the increased coverage of flares in wavelength space and in domains from surface to heliosphere is enabling an improved assessment of energy budgets. For example, Emslie et al. (2012) quantify energies of an ensemble of large, eruptive flares to find, among others that it appears that the energy in accelerated particles during the initial phases of the flare suffice to supply the energy eventually radiated in the flare across the spectrum, and that that total energy is statistically just under the bulk kinetic energy in associated coronal mass ejections.

\subsubsection{Particle acceleration and transport}

The central problem in solar flare theory remains the acceleration of the non-thermal electrons required to explain observed chromospheric HXR sources. Observations with RHESSI and SDO show that coronal electron acceleration can be very efficient; Krucker and Battaglia (2014) find that essentially all electrons in a coronal source of density a few times $10^{9} \mathrm{~cm}^{-3}$ are energized to above around $10 \mathrm{keV}$. Kappa distributions, which are found to be a better fit than a standard thermal plus non-thermal distribution in coronal HXR sources (Oka et al. 2015), are shown to arise naturally in an acceleration region when there is a balance between diffusive acceleration and collisions, in the absence of significant escape from the acceleration region (Bian et al. 2014). In the electron-beam model of a flare, electrons must of course escape the corona to produce the chromospheric HXR sources, and the number flux requirements have always been somewhat 
problematic. This may be alleviated if electrons are boosted by wave-particle interactions in the corona; a quasi-linear simulation of coronal electron propagation shows that waveparticle interaction with the high phase-velocity Langmuir waves generated by density inhomogeneities can accelerate beam electrons to higher energies, reducing the requirement on electron flux at energies of a few tens of $\mathrm{keV}$ by up to a factor ten (Hannah, Kontar, and Reid 2013). Varady et al. (2014) study a model in which electrons are reaccelerated in the chromosphere, concluding that this also reduces demands on putative electron beam fluxes (however requirements on the flare chromosphere energy source, not directly addressed in this model, remain the same). Chromospheric (re-) acceleration models may also produce electron angular distributions which are more isotropic, consistent with the angular distributions inferred from inversion of RHESSI mean electron flux spectra, accounting for photospheric X-ray albedo, by Dickson and Kontar (2013). A completely different view by Melrose and Wheatland (2014) is that the electron energization in flares takes place in a parallel electric field that develops close to or in the chromosphere, in a region of anomalous resistivity, if energy is transported Alfvénically - specifically by inertial Alfvén waves. However, the orthodoxy remains that energy transport is by electron beams, and this is now being tested against observations using beam-driven radiation hydrodynamics codes, the output of which can be compared with, for example, IRIS (Rubio da Costa et al. 2015) and EVE and AIA data (Kennedy et al. 2015), so far with mixed success.

\subsection{Coronal mass ejections}

The understanding of the initiation and evolution of coronal mass ejections (CMEs) has tremendously profited from the combination of coronagraphic observations with high cadence imaging in the EUV together with the multi-perspective view provided by the STEREO mission, as well as from increasingly sophisticated MHD and thermodynamic modeling (for reviews see Webb and Howard 2012; Aulanier 2014). Magnetic flux ropes play a key role in the physics of CMEs. But there is a long debate whether flux ropes are pre-existing or formed during the eruption. Patsourakos, Vourlidas, and Stenborg (2013) observed the formation of a flux rope during a confined flare in high-cadence SDO/AIA EUV imagery. Within hours after its formation, the flux rope became unstable and erupted resulting in a CME/flare event. For other CMEs, for example those associated with quiescent prominence-cavity systems, a variety of observations indicate a pre-existing flux rope which may erupt bodily as a CME (see e.g. Figure 12 of Gibson 2015). Vourlidas et al. (2013) synthesized 16 years of coronagraphic and EUV observations with MHD simulations, and found that flux ropes are a common structure in CMEs; in at least $40 \%$ a clear flux rope structure could be identified. In addition, they established a new "two-front" morphology consisting of a faint front followed by diffuse emission and the bright CME leading edge. The faint front is suggestive of a wave or shock front driven by the CME.

The high-cadence six-passband SDO/AIA EUV imagery allows to perform differential emission measure (DEM) analysis on solar flares and CMEs to study their multi-thermal dynamics (Hannah and Kontar 2012). It was shown that the CME core region, typically identified as the embedded flux rope, is hot $(8-10 \mathrm{MK})$ indicative of magnetic reconnection being involved. In contrast, the CME leading front has temperatures similar to the pre-eruptive corona but of higher densities suggesting that the front is a result of compression of the ambient coronal plasma (Cheng et al. 2012a; Hannah and Kontar 2013). The hot flux rope is a key indicator to the physical processes involved in the early acceleration phase of the CME (Fan 2012; Cheng et al. 2013). 
The environment of CMEs is important for the development of non-radial propagation. Zuccarello et al. (2012) report that during solar minimum conditions CMEs originating from high latitudes can be easily deflected toward the heliospheric current sheet, thus eventually becoming geo-effective. Panasenco et al. (2013) showed that coronal holes nearby the CME initiation site can cause strong deflections of CMEs.

Modeling of the initiation of CMEs continues to provide insights into the various forces and mechanisms that may be involved: initiation may involve the kink instability (Kumar et al. 2012), sunspot rotation, reduction of tension of the overlying field (Török et al. 2013), torus instability (Kliem et al. 2014), and the breakout process (Karpen, Antiochos, and DeVore 2012; Lynch and Edmondson 2013). Which dominates under which conditions and how commonly these occur remain topics of future work.

\subsection{Large-scale EUV waves}

Since their discovery by the SOHO/EIT instrument about 15 years ago, there has been a vivid debate about the physical nature of large-scale EUV waves, i.e. whether they are true wave phenomena or propagating disturbances related to the magnetic restructuring due to the erupting CME. In the recent years, there has been tremendous progress in the understanding of these intriguing phenomena thanks to the unprecedented observations available, in particular the high-cadence EUV imagery in six wavelengths bands by SDO/AIA combined with the STEREO multi-point view which allowed for the first time to follow EUV waves in full-Sun maps (Olmedo et al. 2012). There seems now relatively broad consensus that large-scale EUV waves are often fast-mode magnetosonic waves (of large amplitude or shocks), driven by the strong lateral expansion of the CME (see reviews by Patsourakos and Vourlidas 2012; Liu and Ofman 2014). A number of detailed case studies revealed that the CME lateral front and the EUV wave appear originally co-spatial. But when the lateral CME expansion slows down, the EUV wave decouples from the driver and then propagates freely, adjusting to the local fast-mode speed of the medium (e.g. Cheng et al. 2012b; Olmedo et al. 2012). Three-dimensional thermodynamic MHD modeling of well observed EUV waves also supports these findings, showing the outer fast-mode EUV wave front followed by another bright front indicating the CME component (Downs et al. 2012). Statistical studies of EUV waves based on SDO/AIA (Nitta et al. 2013) and STEREO/EUVI data (Muhr et al. 2014) revealed EUV wave speeds that range from close to the fast magnetosonic speed in the quiet corona to values well above, the fastest ones exceeding $1000 \mathrm{~km} \mathrm{~s}^{-1}$. Muhr et al. (2014) showed that at least half of the EUV waves under study show significant deceleration, and a distinct anti-correlation between the starting speed and the deceleration, providing further evidence for a freely propagating fast-mode wave. The association rate of EUV waves with type II bursts, which are indicative of shock waves in the solar corona, may be as high as $50 \%$ (Nitta et al. 2013). Detailed case studies provided a number of further characteristics suggestive of the wave nature, such as reflection and refraction of EUV waves at coronal holes and active regions, transmission into coronal holes as well as the initiation of secondary waves by the arrival of the wave at structures of high Alfvén speed (Li et al. 2012; Olmedo et al. 2012; Shen and Liu 2012; Kienreich et al. 2013) and for one case, Long et al. (2015) have evaluated the EUV wave's initial energy using a blastwave approximation, to be around $10 \%$ of that of the associated CME. Liu et al. (2012a) discovered quasi-periodic fast-mode wave trains within a large-scale EUV Wave with a periodicity of $2 \mathrm{~min}$, running ahead of the laterally expanding CME flanks. Asai et al. (2012) presented the first simultaneous observations of the propagation of a large-scale EUV wave and an $\mathrm{H} \alpha$ Moreton wave, showing that the wave fronts evolve co-spatially indicating that they are both signatures of a fast magnetosonic wave pulse. 


\subsection{CME evolution in the heliosphere}

The STEREO mission, often in combination with SOHO/LASCO, offers observations of CMEs all the way from their origin on the Sun, and of their propagation in interplanetary space to beyond $1 \mathrm{AU}$ from outside the Sun-Earth line. These data combined with a multitude of other in-situ space missions have been vividly used to connect remote sensing CME observations to the field and plasma data observed by in-situ spacecraft, to constrain models of interplanetary CME propagation, to study CME-CME interaction, and to forecast CME arrival times and speeds with the ultimate aim improving the prediction of their geo-effectiveness.

Howard and DeForest (2012) and DeForest, Howard, and McComas (2013) tracked a flux rope all the way from its solar origin to its in-situ signatures at $1 \mathrm{AU}$ using the STEREO SECCHI EUV imagers, coronagraphs and wide-angle heliospheric imagers. They establish that the cavity in the classic three-part CME is the feature that becomes the magnetic cloud, implying material ahead of the cavity is piled-up material from the corona or the solar wind.

Modeling of the interplanetary propagation of CMEs makes use of empirical, analytic and numerical approaches. The analytical "drag-based model" (DBM) is based on the hypothesis that the Lorentz forces driving a CME eruption ceases in the upper corona and that beyond a certain distance the interplanetary CME (ICME) dynamics is governed solely by the interaction of the ICME and the ambient solar wind plasma (Vršnak et al. 2013). From the observational side, a variety of reconstruction methods have been developed and applied to the Heliospheric Imager data including one- as well as twospacecraft (stereoscopic) observations and inclusion of in-situ data and radio type II bursts to better constrain the propagation direction, distance and speed profile of CMEs in interplanetary space (Rollett et al. 2012; Möstl and Davies 2013; Colaninno, Vourlidas, and $\mathrm{Wu}$ 2013; Liu et al. 2013). These efforts result in comparable typical uncertainties in the CME arrival time of about half a day (Vršnak et al. 2014; Lee et al. 2013; Möstl et al. 2014).

Studies using STEREO Heliospheric Imager data and in-situ plasma and field measurements established that the interaction of CMEs in the inner heliosphere, due to a faster CME launched after a slower one, seems to be a common and important phenomenon (Liu et al. 2012b; Harrison et al. 2012; Lugaz et al. 2012; Temmer et al. 2012; Möstl et al. 2012; Liu et al. 2013; Temmer et al. 2014). The interaction process may cause deflection or merging of CMEs, and either deceleration or acceleration of merged CME fronts (including heating and compression). Liu et al. (2014b), reporting in a fast CME causing an extreme storm, speculate that the interaction between two successively launched CMEs resulted in the extreme enhancement of the magnetic field of the ejecta that was observed in-situ near $1 \mathrm{AU}$.

\subsection{Sun-in-time}

The unusually deep and temporally-extended activity minimum between sunspot cycles 23 and 24, followed by a slowly rising and low amplitude cycle 24, has led to renewed interest in the underlying causes of solar cycle fluctuations, including Grand Minima. Much attention has focused on the so-called Babcock-Leighton solar cycle models, in which the regeneration of the solar surface dipole takes place via the decay of active regions. Most extant versions of these dynamo models are geometrically (axisymmetric) and dynamically (kinematic) simplified, yet they do remarkably well at reproducing many observed solar cycle characteristics (see, e.g., Karak et al. 2014, and references therein). Explanations for the extended cycle 23-24 minimum and low amplitude cycle 24 have been sought in terms of variations in the meridional flow expected to thread the solar 
convection zone (Upton and Hathaway 2014), and patterns of active region emergence and associated feedback on surface flows (Cameron et al. 2014; Jiang et al. 2015). These successes of the Babcock-Leighton modelling framework have however been challenged by helioseismic measurements (Zhao et al. 2013; Schad et al. 2013) indicating that the meridional flow within the convection zone has a far more complex cellular structure than assumed in the majority of these mean-field-like solar cycle models. Possible avenues out of this conundrum are being explored (see, e.g., Hazra et al. 2014; Belucz et al. 2015).

Much effort has also been invested in implementing various form of data assimilation schemes in dynamo models, with the aim of achieving improved forecasting of the amplitude and timing of future sunspot cycles. At this point in time no existing dynamo model-based forecasting scheme has done significantly better than the known precursor skill of the solar surface magnetic dipole moment at times of cycle minima, nonetheless progress is likely forthcoming in this area.

Global magnetohydrodynamical simulations of solar convection have also progressed rapidly in recent years, with many research groups worldwide now running simulations producing large-scale magnetic fields undergoing polarity reversals (e.g. Masada et al. 2013; Nelson et al. 2013; Fan and Fang 2014; Passos and Charbonneau 2014; Warnecke et al. 2014). Due to computing limitations all these simulations run in parameter regimes still far removed from solar interior conditions. Nonetheless, many are producing tantalizingly solar-like features, including rotational torsional oscillations (Beaudoin et al. 2013) equatorward propagation of activity "belts" (Käpylä et al. 2012; Warnecke et al. 2014; Augustson et al. 2015) cyclic in-phase magnetic modulation of convective energy transport (Cossette et al. 2013) and Grand Minima-like interruptions of cyclic behavior (Augustson et al. 2015). One particularly interesting feature is the spontaneous production of magnetic flux tube-like structures within the convection zone, as reported in Nelson et al. (2013). These were found to rise to the top of the simulation domain, partly through magnetic buoyancy, while maintaining their orientation in a manner compatible with Hale' polarity laws (Nelson et al. 2014). This has revived the idea that dynamo action could be wholly contained within the solar convective envelope, rather than relying on the tachocline for the formation and storage of the magnetic flux ropes eventually giving rise to sunspots.

Major efforts have also taken place in reinterpreting and reanalyzing historical observations of magnetic activity. Noteworthy in this respect are the analyses of tilt angle patterns for bipolar magnetic regions (see Pavai et al. 2015, and references therein), and reanalysis of polar faculae data by Munoz-Jaramillo et al. (2012). Of particular importance is the recently completed revision of the international sunspot number (SSN) time series. SSN values for the period 1947-present now account for a discontinuity in the manner of counting spot groups having occurred at the Locarno reference station (Clette et al. 2014). Correcting for this leads to a significant $(\simeq 20 \%)$ decrease in SSN values during the space era. Consequently, reconstructions of solar activity into the distant past using the SSN as a backbone to extrapolate space-borne measurements will need to be reassessed.

Radionuclides generated by the atmospheric impact of galactic cosmic rays (GCRs) provide a crucial proxy for the evolution in the Sun's activity (Usoskin 2008; Potgieter 2013) on time scales longer than a few years or a decade, depending on the radionuclide and its deposition in terrestrial natural archives. New ice core data on ${ }^{10} \mathrm{Be}$ and tree ring data on ${ }^{14} \mathrm{C}$ have been combined to provide better understanding of climate impacts on these records: a joint analysis of composite tree ring data with ice cores from Greenland and Antarctica have enabled the separation of the common signal (assumed to be dominated by solar and heliospheric variability) from terrestrial variability (Steinhilber et al. 
2012). From this, we now have 94 centuries of data on a proxy for solar activity. But translating that proxy into details of solar activity that may drive space weather and terrestrial climate remains a challenge, as reviewed by, e.g. Solanki, Krivova, and Haigh (2013).

\subsection{Developments, discoveries, and surprises}

And then, of course, there were numerous surprising realizations and discoveries, in the real world as much as in the rapidly growing virtual world). We mention merely a small sampling in no particular order: a weak solar cycle following an uncommonly low and long solar minimum (McComas et al. 2013); a series of X-class flares from AR 12192 none of which were associated with a CME, contrasting with statistics to date (Chen et al. 2015; Sun et al. 2015; Thalmann et al. 2015); use of a Sun-grazing comet to probe the high corona and its connection to the innermost heliosphere (Downs et al. 2013; Raymond et al. 2014); an extremely large amount of dense, cool plasma falling back onto the Sun following a massive filament eruption providing a close-up example of distant accretion processes (Reale et al. 2013); reports of enormously energetic flares from what would appear to be Sun-like stars (Nogami et al. 2014) and potential evidence for strong SEP events associated with very energetic solar flaring from ${ }^{14} \mathrm{C}$ records albeit without obvious auroral counterparts (Miyake et al. 2012; Usoskin et al. 2013; Neuhäuser and Neuhäuser 2015); the successful creation of realistic looking sunspots in the computer (Rempel and Cheung 2014); the revision of sunspot numbers that suggests no long-term increase in solar activity occurred over the past few hundred years (Clette et al. 2014); radiative magneto-convective simulations have reached resolution scales of a few kilometers, and suggest comparable energy densities in magnetic and kinetic reservoirs (Rempel 2014); IRIS observations uncovering rapidly-evolving low-lying loops at transition region temperatures, heretofore inferred from emission measure studies but never yet observed (Hansteen et al. 2014); a new model was proposed for coronal heating based on magnetic gradient pumping (Tan 2014); non-potential field models for a continuously-driven corona over a 16-year period was achieved (Yeates 2014); a solar eruption in July of 2012 that would likely have powered a century-level extreme geomagnetic storm, as for the Carrington-Hodgson flare of 1859, had it enveloped Earth (Baker et al. 2013); the realization that Stokes' theorem combined with the induction equation could explain why polar fields should be a good indicator for the strength of the next sunspot cycle (Cameron and Schüssler 2015); the simulation of a sequence of homologous CMEs and demonstration of so-called "canniballistic" behavior (Chatterjee and Fan 2013); the discovery of nested toroidal line-of-sight flows and lagomorphic coronal polarimetric signatures within coronal cavities indicating the presence of magnetic flux ropes (Ba̧k-Stȩślicka et al. 2013); rapidly rotating magnetic structures ("magnetic tornadoes") have been identified, which provide a channel of energy and twist from the solar surface to the corona (Zhang and Liu 2011; Wedemeyer-Böhm et al. 2012); and the X-class flare SOL2014-03-29T made history by becoming "the best-observed flare of all time" (http://www.nasa.gov/content/goddard/nasa-telescopes-coordinate-bestever-flare-observations/\#.VhJ4TrQ9Yow according to NASA) as the ground-based Dunn Solar Telescope and the space-based IRIS, RHESSI, and SDO all observed it in detail.

\section{Acknowledgement}

We gratefully acknowledge making use of NASA's Astrophysics Data System in the writing of this report. 


\section{References}

Al-Ghraibah, A., Boucheron, L. E., \& McAteer, R. T. J. 2015, An automated classification approach to ranking photospheric proxies of magnetic energy build-up. $A \& A$ 579, A64. http://dx.doi.org/10.1051/0004-6361/201525978DOI:10.1051/0004-6361/201525978.

Alvarado-Gómez, J. D., Buitrago-Casas, J. C., Martínez-Oliveros, J. C., Lindsey, C., Hudson, H., \& Calvo-Mozo, B. 2012, Magneto-Acoustic Energetics Study of the Seismically Active Flare of 15 February 2011. Solar Phys. 280, 335. http://dx.doi .org/10.1007/s11207-012-0009-6DOI:10.1007/s11207-012-0009-6.

Amari, T., Canou, A., \& Aly, J.-J. 2014, Characterizing and predicting the magnetic environment leading to solar eruptions. Nature 514, 465. http://dx.doi.org/ 10.1038/nature13815DOI:10.1038/nature13815.

Asai, A., Ishii, T. T., Isobe, H., Kitai, R., Ichimoto, K., UeNo, S., Nagata, S., Morita, S., Nishida, K., Shiota, D., Oi, A., Akioka, M., \& Shibata, K. 2012, First Simultaneous Observation of an $\mathrm{H} \alpha$ Moreton Wave, EUV Wave, and Filament/Prominence Oscillations. Astrophys. J., Lett. 745, L18. http://dx.doi.org/10.1088/2041-8205/745/2/L18DOI:10.1088/2041$8205 / 745 / 2 /$ L 18 .

Aschwanden, M. J., Schrijver, C. J., Malanushenko, A.: 2015, Blind Stereoscopy of the Coronal Magnetic Field. ArXiv e-prints.

Augustson, K., Brun, A. S., Miesch, M., \& Toomre, J. 2015, Grand Minima and Equatorward Propagation in a Cycling Stellar Convective Dynamo. Astrophys. J. 809, 149. http://dx.doi.org/10.1088/0004-637X/809/2/149DOI:10.1088/0004-637X/809/2/149.

Aulanier, G. 2014, The physical mechanisms that initiate and drive solar eruptions. In: Schmieder, B., Malherbe, J.-M., Wu, S. T. (eds.) IAU Symposium, IAU Symposium 300, 184. http://dx.doi.org/10.1017/S1743921313010958DOI:10.1017/S1743921313010958.

Baker, D. N., Li, X., Pulkkinen, A., Ngwira, C. M., Mays, M. L., Galvin, A. B., \& Simunac, K. D. C. 2013, A major solar eruptive event in July 2012: Defining extreme space weather scenarios. Space Weather 11, 585. http://dx.doi.org/ 10.1002/swe.20097DOI:10.1002/swe.20097.

Bastian, T. S., Barta, M., Brajsa, R., Chen, B., De Pontieu, B., Fleishman, G., Gary, D., Hales, A., Hills, R., Hudson, H., Iwai, K., Shimojo, M., White, S., Wedemeyer, S., \& Yan, Y. 2015, The Atacama Large Millimeter/Submillimeter Array: a New Asset for Solar and Heliospheric Physics. IAU General Assembly 22, 57295.

Bạk-Stȩślicka, U., Gibson, S. E., Fan, Y., Bethge, C., Forland, B., \& Rachmeler, L. A. 2013, The Magnetic Structure of Solar Prominence Cavities: New Observational Signature Revealed by Coronal Magnetometry. Astrophys. J., Lett. 770, L28. http://dx.doi.org/10.1088/2041-8205/770/2/L28DOI:10.1088/2041-8205/770/2/L28.

Beaudoin, P., Charbonneau, P., Racine, E., \& Smolarkiewicz, P. K. 2013, Torsional Oscillations in a Global Solar Dynamo. Solar Phys. 282, 335. http://dx.doi.org/ 10.1007/s11207-012-0150-2DOI:10.1007/s11207-012-0150-2.

Belucz, B., Dikpati, M., \& Forgács-Dajka, E. 2015, A Babcock-Leighton Solar Dynamo Model with Multi-cellular Meridional Circulation in Advection- and Diffusion-dominated Regimes. Astrophys. J. 806, 169. http://dx.doi.org/ 10.1088/0004-637X/806/2/169DOI:10.1088/0004-637X/806/2/169.

Bian, N. H., Emslie, A. G., Stackhouse, D. J., \& Kontar, E. P. 2014, The Formation of Kappadistribution Accelerated Electron Populations in Solar Flares. Astrophys. J. 796, 142. http://dx.doi.org/10.1088/0004-637X/796/2/142DOI:10.1088/0004-637X/796/2/142.

Bobra, M. G. \& Couvidat, S. 2015, Solar Flare Prediction Using SDO/HMI Vector Magnetic Field Data with a Machine-learning Algorithm. Astrophys. J. 798, 135. http://dx.doi.org/10.1088/0004-637X/798/2/135DOI:10.1088/0004-637X/798/2/135.

Brannon, S. R., Longcope, D. W., \& Qiu, J. 2015, Spectroscopic Observations of an Evolving Flare Ribbon Substructure Suggesting Origin in Current Sheet Waves. Astrophys. J. 810, 4. http://dx.doi.org/10.1088/0004-637X/810/1/4DOI:10.1088/0004-637X/810/1/4.

Brooks, D. H., Warren, H. P., \& Ugarte-Urra, I. 2012, Solar coronal loops resolved by hinode and the solar dynamics observatory. The Astrophysical Journal Letters 755(2), L33. ISBN 2041-8205. http://stacks.iop.org/2041-8205/755/i=2/a=L33. 
Brosius, J. W. 2013, Chromospheric Evaporation in Solar Flare Loop Strands Observed with the Extreme-ultraviolet Imaging Spectrometer on Board Hinode. Astrophys. J. 762, 133. http://dx.doi.org/10.1088/0004-637X/762/2/133DOI:10.1088/0004-637X/762/2/133.

Brosius, J. W. \& Daw, A. N. 2015, Quasi-periodic Fluctuations and Chromospheric Evaporation in a Solar Flare Ribbon Observed by IRIS. Astrophys. J. 810, 45. http://dx.doi.org/10.1088/0004-637X/810/1/45DOI:10.1088/0004-637X/810/1/45.

Cally, P. S. \& Hansen, S. C. 2011, Benchmarking Fast-to-Alfvén Mode Conversion in a Cold Magnetohydrodynamic Plasma. Astrophys. J. 738, 119. http://dx.doi.org/ 10.1088/0004-637X/738/2/119DOI:10.1088/0004-637X/738/2/119.

Cameron, R. \& Schüssler, M. 2015, The crucial role of surface magnetic fields for the solar dynamo. Science 347, 1333 . http://dx.doi.org/ 10.1126/science.1261470DOI:10.1126/science.1261470.

Cameron, R. H., Jiang, J., Schüssler, M., \& Gizon, L. 2014, Physical causes of solar cycle amplitude variability. Journal of Geophysical Research (Space Physics) 119, 680. http://dx.doi.org/10.1002/2013JA019498DOI:10.1002/2013JA019498.

Chatterjee, P. \& Fan, Y. 2013, Simulation of Homologous and Cannibalistic Coronal Mass Ejections produced by the Emergence of a Twisted Flux Rope into the Solar Corona. Astrophys. J., Lett. 778, L8. http://dx.doi.org/10.1088/2041-8205/778/1/L8DOI:10.1088/2041$8205 / 778 / 1 /$ L8.

Chen, B., Bastian, T. S., White, S. M., Gary, D. E., Perley, R., Rupen, M., \& Carlson, B. 2013, Tracing Electron Beams in the Sun's Corona with Radio Dynamic Imaging Spectroscopy. Astrophys. J., Lett. 763, L21. http://dx.doi.org/ 10.1088/2041-8205/763/1/L21DOI:10.1088/2041-8205/763/1/L21.

Chen, H., Zhang, J., Ma, S., Yang, S., Li, L., Huang, X., \& Xiao, J. 2015, Confined Flares in Solar Active Region 12192 from 2014 October 18 to 29. Astrophys. J., Lett. 808, L24. http://dx.doi.org/10.1088/2041-8205/808/1/L24DOI:10.1088/2041-8205/808/1/L24.

Cheng, X., Zhang, J., Saar, S. H., \& Ding, M. D. 2012a, Differential Emission Measure Analysis of Multiple Structural Components of Coronal Mass Ejections in the Inner Corona. Astrophys. J. 761, 62. http://dx.doi.org/10.1088/0004-637X/761/1/62DOI:10.1088/0004$637 \mathrm{X} / 761 / 1 / 62$.

Cheng, X., Zhang, J., Olmedo, O., Vourlidas, A., Ding, M. D., \& Liu, Y. 2012b, Investigation of the Formation and Separation of an Extreme-ultraviolet Wave from the Expansion of a Coronal Mass Ejection. Astrophys. J., Lett. 745, L5. http://dx.doi.org/10.1088/2041-8205/745/1/L5DOI:10.1088/2041-8205/745/1/L5.

Cheng, X., Zhang, J., Ding, M. D., Olmedo, O., Sun, X. D., Guo, Y., \& Liu, Y. 2013, Investigating Two Successive Flux Rope Eruptions in a Solar Active Region. Astrophys. J., Lett. 769, L25. http://dx.doi.org/10.1088/2041-8205/769/2/L25DOI:10.1088/2041$8205 / 769 / 2 /$ L25.

Cheung, M. C. M. \& DeRosa, M. L. 2012, A Method for Data-driven Simulations of Evolving Solar Active Regions. Astrophys. J. 757, 147. http://dx.doi.org/ 10.1088/0004-637X/757/2/147DOI:10.1088/0004-637X/757/2/147.

Cirtain, J. W., Golub, L., Winebarger, A. R., De Pontieu, B., Kobayashi, K., Moore, R. L., Walsh, R. W., Korreck, K. E., Weber, M., McCauley, P., Title, A., Kuzin, S., \& DeForest, C. E. 2013, Energy release in the solar corona from spatially resolved magnetic braids. Nature 493(7433), 501. ISBN 0028-0836. http://dx.doi.org/10.1038/nature11772.

Clette, F., Svalgaard, L., Vaquero, J. M., \& Cliver, E. W. 2014, Revisiting the Sunspot Number. A 400-Year Perspective on the Solar Cycle. Space Sci. Rev. 186, 35. http://dx.doi.org/10.1007/s11214-014-0074-2DOI:10.1007/s11214-014-0074-2.

Colaninno, R. C., Vourlidas, A., \& Wu, C. C. 2013, Quantitative comparison of methods for predicting the arrival of coronal mass ejections at Earth based on multiview imaging. Journal of Geophysical Research (Space Physics) 118, 6866. http://dx.doi.org/10.1002/2013JA019205DOI:10.1002/2013JA019205.

Cossette, J. -F., Charbonneau, P., \& Smolarkiewicz, P. K. 2013, Cyclic Thermal Signature in a Global MHD Simulation of Solar Convection. Astrophys. J., Lett. 777, L29. http://dx.doi.org/10.1088/2041-8205/777/2/L29DOI:10.1088/2041-8205/777/2/L29. 
Cranmer, S. R. \& van Ballegooijen, A. A. 2005, On the Generation, Propagation, and Reflection of Alfvén Waves from the Solar Photosphere to the Distant Heliosphere. Astrophys. J., Suppl. Ser. 156, 265. http://dx.doi.org/10.1086/426507DOI:10.1086/426507.

Culhane, L., Harra, L. K., Baker, D., van Driel-Gesztelyi, L., Sun, J., Doschek, G. A., Brooks, D. H., Lundquist, L. L., Kamio, S., Young, P. R., \& Hansteen, V. H. 2007, Hinode euv study of jets in the suns south polar corona. Publications of the Astronomical Society of Japan 59(sp3), S751. http://dx.doi.org/ 10.1093/pasj/59.sp3.S751DOI:10.1093/pasj/59.sp3.S751. http://pasj.oxfordjournals.org/ content $/ 59 / \mathrm{sp} 3 / \mathrm{S} 751$.abstract.

De Moortel, I. \& Nakariakov, V. M. 2012, Magnetohydrodynamic waves and coronal seismology: an overview of recent results. Royal Society of London Philosophical Transactions Series A 370, 3193. http://dx.doi.org/10.1098/rsta.2011.0640DOI:10.1098/rsta.2011.0640.

De Moortel, I. \& Pascoe, D. J. 2012, The Effects of Line-of-sight Integration on Multistrand Coronal Loop Oscillations. Astrophys. J. 746, 31. http://dx.doi.org/10.1088/0004-637X/746/1/31DOI:10.1088/0004-637X/746/1/31.

De Moortel, I., McIntosh, S. W., Threlfall, J., Bethge, C., \& Liu, J. 2014, Potential Evidence for the Onset of Alfvénic Turbulence in Trans-equatorial Coronal Loops. Astrophys. J., Lett. 782, L34. http://dx.doi.org/10.1088/2041-8205/782/2/L34DOI:10.1088/20418205/782/2/L34.

De Pontieu, B., Rouppe van der Voort, L., McIntosh, S. W., Pereira, T. M. D., Carlsson, M., Hansteen, V., Skogsrud, H., Lemen, J., Title, A., Boerner, P., Hurlburt, N., Tarbell, T. D., Wuelser, J. P., De Luca, E. E., Golub, L., McKillop, S., Reeves, K., Saar, S., Testa, P., Tian, H., Kankelborg, C., Jaeggli, S., Kleint, L., Martinez-Sykora, J.: 2014, On the prevalence of small-scale twist in the solar chromosphere and transition region. Science 346(6207).

De Pontieu, B., Rouppe van der Voort, L., McIntosh, S. W., Pereira, T. M. D., Carlsson, M., Hansteen, V., Skogsrud, H., Lemen, J., Title, A., Boerner, P., Hurlburt, N., Tarbell, T. D., Wuelser, J. P., De Luca, E. E., Golub, L., McKillop, S., Reeves, K., Saar, S., Testa, P., Tian, H., Kankelborg, C., Jaeggli, S., Kleint, L., \& Martinez-Sykora, J. 2014a, On the prevalence of small-scale twist in the solar chromosphere and transition region. Science 346, 1255732. http://dx.doi.org/10.1126/science.1255732DOI:10.1126/science.1255732.

De Pontieu, B., Title, A. M., Lemen, J. R., Kushner, G. D., Akin, D. J., Allard, B., Berger, T., Boerner, P., Cheung, M., Chou, C., Drake, J. F., Duncan, D. W., Freeland, S., Heyman, G. F., Hoffman, C., Hurlburt, N. E., Lindgren, R. W., Mathur, D., Rehse, R., Sabolish, D., Seguin, R., Schrijver, C. J., Tarbell, T. D., Wülser, J.-P., Wolfson, C. J., Yanari, C., Mudge, J., Nguyen-Phuc, N., Timmons, R., van Bezooijen, R., Weingrod, I., Brookner, R., Butcher, G., Dougherty, B., Eder, J., Knagenhjelm, V., Larsen, S., Mansir, D., Phan, L., Boyle, P., Cheimets, P. N., DeLuca, E. E., Golub, L., Gates, R., Hertz, E., McKillop, S., Park, S., Perry, T., Podgorski, W. A., Reeves, K., Saar, S., Testa, P., Tian, H., Weber, M., Dunn, C., Eccles, S., Jaeggli, S. A., Kankelborg, C. C., Mashburn, K., Pust, N., Springer, L., Carvalho, R., Kleint, L., Marmie, J., Mazmanian, E., Pereira, T. M. D., Sawyer, S., Strong, J., Worden, S. P., Carlsson, M., Hansteen, V. H., Leenaarts, J., Wiesmann, M., Aloise, J., Chu, K.-C., Bush, R. I., Scherrer, P. H., Brekke, P., Martinez-Sykora, J., Lites, B. W., McIntosh, S. W., Uitenbroek, H., Okamoto, T. J., Gummin, M. A., Auker, G., Jerram, P., Pool, P., \& Waltham, N. 2014b, The Interface Region Imaging Spectrograph (IRIS). Solar Phys. 289, 2733. http://dx.doi.org/10.1007/s11207-014-0485-yDOI:10.1007/s11207-014-0485-y.

DeForest, C. E., Howard, T. A., \& McComas, D. J. 2013, Tracking Coronal Features from the Low Corona to Earth: A Quantitative Analysis of the 2008 December 12 Coronal Mass Ejection. Astrophys. J. 769, 43. http://dx.doi.org/ 10.1088/0004-637X/769/1/43DOI:10.1088/0004-637X/769/1/43.

Deng, N., Tritschler, A., Jing, J., Chen, X., Liu, C., Reardon, K., Denker, C., $\mathrm{Xu}$, Y., \& Wang, H. 2013, High-cadence and High-resolution $\mathrm{H} \alpha$ Imaging Spectroscopy of a Circular Flare's Remote Ribbon with IBIS. Astrophys. J. 769, 112. http://dx.doi.org/10.1088/0004-637X/769/2/112DOI:10.1088/0004-637X/769/2/112.

DeRosa, M. L., Wheatland, M. S., Leka, K. D., Barnes, G., Amari, T., Canou, A., Gilchrist, S. A., Thalmann, J. K., Valori, G., Wiegelmann, T., Schrijver, C. J., Malanushenko, A., Sun, X., \& Régnier, S. 2015, The Influence of Spatial Resolution on Nonlinear Force-Free Modeling. Solar Phys. in press. 
DeRosa, M. L., Schrijver, C. J., Barnes, G., Leka, K. D., Lites, B. W., Aschwanden, M. J., Amari, T., Canou, A., McTiernan, J. M., Regnier, S., Thalmann, J. K., Valori, G., Wheatland, M. S., Wiegelmann, T., Cheung, M. C. M., Conlon, P. A., Fuhrmann, M., Inhester, B., \& Tadesse, T. 2009, A critical assessment of the feasibility of nonlinear force-free field modeling of the solar corona. Astrophys. J. 696, 1780.

Dickson, E. C. M., \& Kontar, E. P. 2013, Measurements of Electron Anisotropy in Solar Flares Using Albedo with RHESSI X-Ray Data. Solar Phys. 284, 405. http://dx.doi.org/10.1007/s11207-012-0178-3DOI:10.1007/s11207-012-0178-3.

Downs, C., Roussev, I. I., van der Holst, B., Lugaz, N., \& Sokolov, I. V. 2012, Understanding SDO/AIA Observations of the 2010 June 13 EUV Wave Event: Direct Insight from a Global Thermodynamic MHD Simulation. Astrophys. J. 750, 134.

Downs, C., Linker, J. A., Mikić, Z., Riley, P., Schrijver, C. J., \& Saint-Hilaire, P. 2013, Probing the Solar Magnetic Field with a Sun-Grazing Comet. Science 340, 1196. http://dx.doi.org/10.1126/science.1236550DOI:10.1126/science.1236550.

Emslie, A. G., Dennis, B. R., Shih, A. Y., Chamberlin, P. C., Mewaldt, R. A., Moore, C. S., Share, G. H., Vourlidas, A., \& Welsch, B. T. 2012, Global Energetics of Thirty-eight Large Solar Eruptive Events. Astrophys. J. 759, 71. http://dx.doi.org/ 10.1088/0004-637X/759/1/71DOI:10.1088/0004-637X/759/1/71.

Fan, Y. 2012, Thermal Signatures of Tether-cutting Reconnections in Pre-eruption Coronal Flux Ropes: Hot Central Voids in Coronal Cavities. Astrophys. J. 758, 60. http://dx.doi.org/10.1088/0004-637X/758/1/60DOI:10.1088/0004-637X/758/1/60.

Fan, Y. \& Fang, F. 2014, A Simulation of Convective Dynamo in the Solar Convective Envelope: Maintenance of the Solar-like Differential Rotation and Emerging Flux. Astrophys. J. 789, 35. http://dx.doi.org/10.1088/0004-637X/789/1/35DOI:10.1088/0004$637 \mathrm{X} / 789 / 1 / 35$.

Fisher, G. H., Abbett, W. P., Bercik, D. J., Kazachenko, M. D., Lynch, B. J., Welsch, B. T., Hoeksema, J. T., Hayashi, K., Liu, Y., Norton, A. A., Dalda, A. S., Sun, X., DeRosa, M. L., \& Cheung, M. C. M. 2015, The Coronal Global Evolutionary Model: Using HMI Vector Magnetogram and Doppler Data to Model the Buildup of Free Magnetic Energy in the Solar Corona. Space Weather 13, 369. http://dx.doi.org/10.1002/2015SW001191DOI:10.1002/2015SW001191.

Giacconi, R., Reidy, W. P., Zehnpfennig, T., Lindsay, J. C., \& Muney, W. S. 1965, Solar X-Ray Image Obtained Using Grazing-Incidence Optics. Astrophys. J. 142, 1274. http://dx.doi.org/10.1086/148404DOI:10.1086/148404.

Gibson, S. 2015, Coronal Cavities: Observations and Implications for the Magnetic Environment of Prominences. In: Vial, J.-C., Engvold, O. (eds.) Astrophysics and Space Science Library, Astrophysics and Space Science Library 415, 323.

Gómez, D. O. 2010, Heating of coronal active regions. In: The Physics of Sun and Star Spots, Proceedings of the International Astronomical Union 6, 44. http://dx.doi.org/10.1017/S1743921311014980DOI:10.1017/S1743921311014980.

Gómez, D. O., Martens, P. C. H., \& Golub, L. 1993, Normal incidence X-ray telescope power spectra of X-ray emission from solar active regions. I - Observations. II - Theory. Astrophys. J. 405, 767. http://dx.doi.org/10.1086/172405DOI:10.1086/172405.

Goode, P. R., \& Cao, W. 2012, The $1.6 \mathrm{~m}$ off-axis New Solar Telescope (NST) in Big Bear. In: Society of Photo-Optical Instrumentation Engineers (SPIE) Conference Series, Society of Photo-Optical Instrumentation Engineers (SPIE) Conference Series 8444, 3. http://dx.doi.org/10.1117/12.925494DOI:10.1117/12.925494.

Graham, D. R. \& Cauzzi, G. 2015, Temporal Evolution of Multiple Evaporating Ribbon Sources in a Solar Flare. Astrophys. J., Lett. 807, L22. http://dx.doi.org/ 10.1088/2041-8205/807/2/L22DOI:10.1088/2041-8205/807/2/L22.

Graham, D. R., Hannah, I. G., Fletcher, L., \& Milligan, R. O. 2013, The Emission Measure Distribution of Impulsive Phase Flare Footpoints. Astrophys. J. 767, 83. http://dx.doi.org/10.1088/0004-637X/767/1/83DOI:10.1088/0004-637X/767/1/83.

Hannah, I. G., \& Kontar, E. P. 2012, Differential emission measures from the regularized inversion of Hinode and SDO data. $A \& A$ A 539, A146. http://dx.doi.org/ 10.1051/0004-6361/201117576DOI:10.1051/0004-6361/201117576. 
Hannah, I. G., \& Kontar, E. P. 2013, Multi-thermal dynamics and energetics of a coronal mass ejection in the low solar atmosphere. A\&A 553, A10. http://dx.doi.org/ 10.1051/0004-6361/201219727DOI:10.1051/0004-6361/201219727.

Hannah, I. G., Kontar, E. P., \& Reid, H. A. S. 2013, Effect of turbulent densityfluctuations on wave-particle interactions and solar flare X-ray spectra. A $B A$ 550, A51. http://dx.doi.org/10.1051/0004-6361/201220462DOI:10.1051/0004-6361/201220462.

Hansen, S. C., \& Cally, P. S. 2012, Benchmarking Fast-to-Alfvén Mode Conversion in a Cold MHD Plasma. II. How to get Alfvén waves through the Solar Transition Region. Astrophys. J. 751, 31. http://dx.doi.org/doi:10.1088/0004-637X/751/1/31DOI:doi:10.1088/0004$637 \mathrm{X} / 751 / 1 / 31$.

Hansteen, V., De Pontieu, B., Carlsson, M., Lemen, J., Title, A., Boerner, P., Hurlburt, N., Tarbell, T. D., Wuelser, J. P., Pereira, T. M. D., De Luca, E. E., Golub, L., McKillop, S., Reeves, K., Saar, S., Testa, P., Tian, H., Kankelborg, C., Jaeggli, S., Kleint, L., \& Martínez-Sykora, J. 2014, The unresolved fine structure resolved: IRIS observations of the solar transition region. Science 346. http://dx.doi.org/10.1126/science.1255757DOI:10.1126/science.1255757.

Harrison, R. A., Davies, J. A., Möstl, C., Liu, Y., Temmer, M., Bisi, M. M., Eastwood, J. P., de Koning, C. A., Nitta, N., Rollett, T., Farrugia, C. J., Forsyth, R. J., Jackson, B. V., Jensen, E. A., Kilpua, E. K. J., Odstrcil, D., \& Webb, D. F. 2012, An Analysis of the Origin and Propagation of the Multiple Coronal Mass Ejections of 2010 August 1. Astrophys. J. 750, 45.

Hayashi, K., Hoeksema, J. T., Liu, Y., Bobra, M. G., Sun, X. D., \& Norton, A. A. 2015, The Helioseismic and Magnetic Imager (HMI) Vector Magnetic Field Pipeline: Magnetohydrodynamics Simulation Module for the Global Solar Corona. Solar Phys. 290, 1507. http://dx.doi.org/10.1007/s11207-015-0686-zDOI:10.1007/s11207-015-0686-z.

Hazra, G., Karak, B. B., \& Choudhuri, A. R. 2014, Is a Deep One-cell Meridional Circulation Essential for the Flux Transport Solar Dynamo? Astrophys. J. 782, 93. http://dx.doi.org/10.1088/0004-637X/782/2/93DOI:10.1088/0004-637X/782/2/93.

Heinzel, P. \& Kleint, L. 2014, Hydrogen Balmer Continuum in Solar Flares Detected by the Interface Region Imaging Spectrograph (IRIS). Astrophys. J., Lett. 794, L23. http://dx.doi.org/10.1088/2041-8205/794/2/L23DOI:10.1088/2041-8205/794/2/L23.

Howard, T. A., \& DeForest, C. E. 2012, Inner Heliospheric Flux Rope Evolution via Imaging of Coronal Mass Ejections. Astrophys. J. 746, 64. http://dx.doi.org/ 10.1088/0004-637X/746/1/64DOI:10.1088/0004-637X/746/1/64.

Jiang, J., Cameron, R. H., \& Schüssler, M. 2015, The Cause of the Weak Solar Cycle 24. Astrophys. J., Lett. 808, L28. http://dx.doi.org/ 10.1088/2041-8205/808/1/L28DOI:10.1088/2041-8205/808/1/L28.

Kaiser, M. L., Kucera, T. A., Davila, J. M., St. Cyr, O. C., Guhathakurta, M., \& Christian, E. 2008, The STEREO Mission: An Introduction. Space Science Reviews 136, 5. http://dx.doi.org/10.1007/s11214-007-9277-0DOI:10.1007/s11214-007-9277-0.

Käpylä, P. J., Mantere, M. J., \& Brandenburg, A. 2012, Cyclic Magnetic Activity due to Turbulent Convection in Spherical Wedge Geometry. Astrophys. J., Lett. 755, L22. http://dx.doi.org/10.1088/2041-8205/755/1/L22DOI:10.1088/2041-8205/755/1/L22.

Karak, B. B., Jiang, J., Miesch, M. S., Charbonneau, P., \& Choudhuri, A. R. 2014, Flux Transport Dynamos: From Kinematics to Dynamics. Space Sci. Rev. 186, 561. http://dx.doi.org/10.1007/s11214-014-0099-6DOI:10.1007/s11214-014-0099-6.

Karpen, J. T., Antiochos, S. K., \& DeVore, C. R. 2012, The Mechanisms for the Onset and Explosive Eruption of Coronal Mass Ejections and Eruptive Flares. Astrophys. J. 760, 81. http://dx.doi.org/10.1088/0004-637X/760/1/81DOI:10.1088/0004-637X/760/1/81.

Kennedy, M. B., Milligan, R. O., Allred, J. C., Mathioudakis, M., \& Keenan, F. P. 2015, Radiative hydrodynamic modelling and observations of the X-class solar flare on 2011 March 9. A\&A 578, A72. http://dx.doi.org/10.1051/0004-6361/201425144DOI:10.1051/0004$6361 / 201425144$.

Kerr, G. S. \& Fletcher, L. 2014, Physical Properties of White-light Sources in the 2011 February 15 Solar Flare. Astrophys. J. 783, 98. http://dx.doi.org/ 10.1088/0004-637X/783/2/98DOI:10.1088/0004-637X/783/2/98.

Kienreich, I. W., Muhr, N., Veronig, A. M., Berghmans, D., De Groof, A., Temmer, M., Vršnak, B., \& Seaton, D. B. 2013, Solar TErrestrial Relations Observatory-A 
(STEREO-A) and PRoject for On-Board Autonomy 2 (PROBA2) Quadrature Observations of Reflections of Three EUV Waves from a Coronal Hole. Solar Phys. 286, 201. http://dx.doi.org/10.1007/s11207-012-0023-8DOI:10.1007/s11207-012-0023-8.

Kliem, B., Lin, J., Forbes, T. G., Priest, E. R., \& Török, T. 2014, Catastrophe versus Instability for the Eruption of a Toroidal Solar Magnetic Flux Rope. Astrophys. J. 789, 46. http://dx.doi.org/10.1088/0004-637X/789/1/46DOI:10.1088/0004-637X/789/1/46.

Kosugi, T., Matsuzaki, K., Sakao, T., Shimizu, T., Sone, Y., Tachikawa, S., Hashimoto, T., Minesugi, K., Ohnishi, A., Yamada, T., Tsuneta, S., Hara, H., Ichimoto, K., Suematsu, Y., Shimojo, M., Watanabe, T., Shimada, S., Davis, J. M., Hill, L. D., Owens, J. K., Title, A. M., Culhane, J. L., Harra, L. K., Doschek, G. A., \& Golub, L. 2007, The Hinode (Solar-B) mission: an overview. Solar Phys. 243, 1.

Krucker, S., \& Battaglia, M. 2014, Particle Densities within the Acceleration Region of a Solar Flare. Astrophys. J. 780, 107. http://dx.doi.org/ 10.1088/0004-637X/780/1/107DOI:10.1088/0004-637X/780/1/107.

Krucker, S., Saint-Hilaire, P., Hudson, H. S., Haberreiter, M., Martinez-Oliveros, J. C., Fivian, M. D., Hurford, G., Kleint, L., Battaglia, M., Kuhar, M., \& Arnold, N. G. 2015, Co-Spatial White Light and Hard X-Ray Flare Footpoints Seen Above the Solar Limb. Astrophys. J. 802, 19. http://dx.doi.org/10.1088/0004-637X/802/1/19DOI:10.1088/0004$637 \mathrm{X} / 802 / 1 / 19$.

Kumar, P., Cho, K.-S., Bong, S.-C., Park, S.-H., \& Kim, Y. H. 2012, Initiation of Coronal Mass Ejection and Associated Flare Caused by Helical Kink Instability Observed by SDO/AIA. Astrophys. J. 746, 67. http://dx.doi.org/ 10.1088/0004-637X/746/1/67DOI:10.1088/0004-637X/746/1/67.

Lee, C. O., Arge, C. N., Odstrčil, D., Millward, G., Pizzo, V., Quinn, J. M., \& Henney, C. J. 2013, Ensemble Modeling of CME Propagation. Solar Phys. 285, 349. http://dx.doi.org/10.1007/s11207-012-9980-1DOI:10.1007/s11207-012-9980-1.

Leibacher, J., Sakurai, T., Schrijver, C. J., \& van Driel-Gesztelyi, L. 2010, Solar Observation Target Identification Convention for use in Solar Physics. Solar Phys. 263, 1.

Lemen, J. R., Title, A. M., Akin, D. J., Boerner, P. F., Chou, C., Drake, J. F., Duncan, D. W., Edwards, C. G., Friedlaender, F. M., Heyman, G. F., Hurlburt, N. E., Katz, N. L., Kushner, G. D., Levay, M., Lindgren, R. W., Mathur, D. P., McFeaters, E. L., Mitchell, S., Rehse, R. A., Schrijver, C. J., Springer, L. A., Stern, R. A., Tarbell, T. D., Wuelser, J.-P., Wolfson, C. J., Yanari, C., Bookbinder, J. A., Cheimets, P. N., Caldwell, D., Deluca, E. E., Gates, R., Golub, L., Park, S., Podgorski, W. A., Bush, R. I., Scherrer, P. H., Gummin, M. A., Smith, P., Auker, G., Jerram, P., Pool, P., Soufli, R., Windt, D. L., Beardsley, S., Clapp, M., Lang, J., \& Waltham, N. 2012, The Atmospheric Imaging Assembly (AIA) on the Solar Dynamics Observatory (SDO). Solar Phys. 275, 17.

Li, T., Zhang, J., Yang, S., \& Liu, W. 2012, SDO/AIA Observations of Secondary Waves Generated by Interaction of the 2011 June 7 Global EUV Wave with Solar Coronal Structures. Astrophys. J. 746, 13. http://dx.doi.org/10.1088/0004-637X/746/1/13DOI:10.1088/0004$637 \mathrm{X} / 746 / 1 / 13$.

Lin, R. P., Dennis, B. R., Hurford, G. J., Smith, D. M., Zehnder, A., Harvey, P. R., Curtis, D. W., Pankow, D., Turin, P., Bester, M., Csillaghy, A., Lewis, M., Madden, N., van Beek, H. F., Appleby, M., Raudorf, T., McTiernan, J., Ramaty, R., Schmahl, E., Schwartz, R., Krucker, S., Abiad, R., Quinn, T., Berg, P., Hashii, M., Sterling, R., Jackson, R., Pratt, R., Campbell, R. D., Malone, D., Landis, D., Barrington-Leigh, C. P., Slassi-Sennou, S., Cork, C., Clark, D., Amato, D., Orwig, L., Boyle, R., Banks, I. S., Shirey, K., Tolbert, A. K., Zarro, D., Snow, F., Thomsen, K., Henneck, R., McHedlishvili, A., Ming, P., Fivian, M., Jordan, J., Wanner, R., Crubb, J., Preble, J., Matranga, M., Benz, A., Hudson, H., Canfield, R. C., Holman, G. D., Crannell, C., Kosugi, T., Emslie, A. G., Vilmer, N., Brown, J. C., Johns-Krull, C., Aschwanden, M., Metcalf, T., \& Conway, A. 2002, The Reuven Ramaty High-Energy Solar Spectroscopic Imager (RHESSI). Solar Phys. 210, 3. http://dx.doi.org/10.1023/A:1022428818870DOI:10.1023/A:1022428818870.

Liu, J., McIntosh, S. W., De Moortel, I., Threlfall, J., \& Bethge, C. 2014a, Statistical Evidence for the Existence of Alfvénic Turbulence in Solar Coronal Loops. Astrophys. J. 797, 7. http://dx.doi.org/10.1088/0004-637X/797/1/7DOI:10.1088/0004-637X/797/1/7.

Liu, W., \& Ofman, L. 2014, Advances in Observing Various Coronal EUV Waves in the SDO Era and Their Seismological Applications (Invited Review). Solar Phys. 289, 3233. 
Liu, W., Ofman, L., Nitta, N. V., Aschwanden, M. J., Schrijver, C. J., Title, A. M., \& Tarbell, T. D. 2012a, Quasi-periodic Fast-mode Wave Trains within a Global EUV Wave and Sequential Transverse Oscillations Detected by SDO/AIA. Astrophys. J. 753, 52.

Liu, Y. D., Luhmann, J. G., Möstl, C., Martinez-Oliveros, J. C., Bale, S. D., Lin, R. P., Harrison, R. A., Temmer, M., Webb, D. F., \& Odstrcil, D. 2012b, Interactions between Coronal Mass Ejections Viewed in Coordinated Imaging and in situ Observations. Astrophys. J., Lett. 746, L15. http://dx.doi .org/10.1088/2041-8205/746/2/L15DOI:10.1088/2041$8205 / 746 / 2 / \mathrm{L} 15$.

Liu, Y. D., Luhmann, J. G., Lugaz, N., Möstl, C., Davies, J. A., Bale, S. D., \& Lin, R. P. 2013, On Sun-to-Earth Propagation of Coronal Mass Ejections. Astrophys. J. 769, 45. http://dx.doi.org/10.1088/0004-637X/769/1/45DOI:10.1088/0004-637X/769/1/45.

Liu, Y. D., Luhmann, J. G., Kajdič, P., Kilpua, E. K. J., Lugaz, N., Nitta, N. V., Möstl, C., Lavraud, B., Bale, S. D., Farrugia, C. J., \& Galvin, A. B. 2014b, Observations of an extreme storm in interplanetary space caused by successive coronal mass ejections. Nature Communications 5, 3481. http://dx.doi.org/10.1038/ncomms4481DOI:10.1038/ncomms4481.

Liu, Z., Xu, J., Gu, B.-Z., Wang, S., You, J.-Q., Shen, L.-X., Lu, R.-W., Jin, Z.-Y., Chen, L.-F., Lou, K., Li, Z., Liu, G.-Q., Xu, Z., Rao, C.-H., Hu, Q.-Q., Li, R.-F., Fu, H.-W., Wang, F., Bao, M.-X., Wu, M.-C., \& Zhang, B.-R. 2014c, New vacuum solar telescope and observations with high resolution. Research in Astronomy and Astrophysics 14, 705. http://dx.doi.org/10.1088/1674-4527/14/6/009DOI:10.1088/1674-4527/14/6/009.

Long, D. M., Baker, D., Williams, D. R., Carley, E. P., Gallagher, P. T., \& Zucca, P. 2015, The Energetics of a Global Shock Wave in the Low Solar Corona. Astrophys. J. 799, 224. http://dx.doi.org/10.1088/0004-637X/799/2/224DOI:10.1088/0004-637X/799/2/224.

Lugaz, N., Farrugia, C. J., Davies, J. A., Möstl, C., Davis, C. J., Roussev, I. I., \& Temmer, M. 2012, The Deflection of the Two Interacting Coronal Mass Ejections of 2010 May 23-24 as Revealed by Combined in Situ Measurements and Heliospheric Imaging. Astrophys. J. 759, 68. http://dx.doi.org/10.1088/0004-637X/759/1/68DOI:10.1088/0004$637 \mathrm{X} / 759 / 1 / 68$.

Lynch, B. J., \& Edmondson, J. K. 2013, Sympathetic Magnetic Breakout Coronal Mass Ejections from Pseudostreamers. Astrophys. J. 764, 87.

Malanushenko, A., Schrijver, C. J., DeRosa, M. L., \& Wheatland, M. S. 2014, Using Coronal Loops to Reconstruct the Magnetic Field of an Active Region before and after a Major Flare. Astrophys. J. 783, 102. http://dx.doi.org/ 10.1088/0004-637X/783/2/102DOI:10.1088/0004-637X/783/2/102.

Mann, G., Vocks, C., \& Breitling, F. 2011, Solar Observations with LOFAR. Planetary, Solar and Heliospheric Radio Emissions (PRE VII), 507.

Martínez Oliveros, J.-C., Hudson, H. S., Hurford, G. J., Krucker, S., Lin, R. P., Lindsey, C., Couvidat, S., Schou, J., \& Thompson, W. T. 2012, The Height of a White-light Flare and Its Hard X-Ray Sources. Astrophys. J., Lett. 753, L26. http://dx.doi.org/10.1088/2041-8205/753/2/L26DOI:10.1088/2041-8205/753/2/L26.

Masada, Y., Yamada, K., \& Kageyama, A. 2013, Effects of Penetrative Convection on Solar Dynamo. Astrophys. J. 778, 11. http://dx.doi.org/ 10.1088/0004-637X/778/1/11DOI:10.1088/0004-637X/778/1/11.

Mathioudakis, M., Jess, D. B., \& Erdélyi, R. 2013, Alfvén Waves in the Solar Atmosphere. From Theory to Observations. Space Sci. Rev. 175, 1. http://dx.doi.org/ 10.1007/s11214-012-9944-7DOI:10.1007/s11214-012-9944-7.

McComas, D. J., Angold, N., Elliott, H. A., Livadiotis, G., Schwadron, N. A., Skoug, R. M., \& Smith, C. W. 2013, Weakest Solar Wind of the Space Age and the Current "Mini" Solar Maximum. Astrophys. J. 779, 2. http://dx.doi.org/ 10.1088/0004-637X/779/1/2DOI:10.1088/0004-637X/779/1/2.

McIntosh, S. W., \& De Pontieu, B. 2012, Estimating the "Dark" Energy Content of the Solar Corona. Astrophys. J. 761, 138. http://dx.doi.org/ 10.1088/0004-637X/761/2/138DOI:10.1088/0004-637X/761/2/138.

McIntosh, S. W., de Pontieu, B., Carlsson, M., Hansteen, V., Boerner, P., \& Goossens, M. 2011, Alfvénic waves with sufficient energy to power the quiet solar corona and fast solar wind. Nature 475, 477. http://dx.doi.org/10.1038/nature10235DOI:10.1038/nature10235. 
Melrose, D. B., \& Wheatland, M. S. 2014, Bulk Energization of Electrons in Solar Flares by Alfvén Waves. Solar Phys. 289, 881. http://dx.doi.org/ 10.1007/s11207-013-0376-7DOI:10.1007/s11207-013-0376-7.

Miyake, F., Nagaya, K., Masuda, K., \& Nakamura, T. 2012, A signature of cosmic-ray increase in AD 774-775 from tree rings in Japan. Nature 486, 240. http://dx.doi.org/ 10.1038/nature11123DOI:10.1038/nature11123.

Morton, R. J., Tomczyk, S., \& Pinto, R. 2015, Investigating Alfvénic wave propagation in coronal open-field regions. Nature Communications 6, 7813. http://dx.doi.org/10.1038/ncomms8813DOI:10.1038/ncomms8813.

Möstl, C., \& Davies, J. A. 2013, Speeds and Arrival Times of Solar Transients Approximated by Self-similar Expanding Circular Fronts. Solar Phys. 285, 411. http://dx.doi.org/10.1007/s11207-012-9978-8DOI:10.1007/s11207-012-9978-8.

Möstl, C., Farrugia, C. J., Kilpua, E. K. J., Jian, L. K., Liu, Y., Eastwood, J. P., Harrison, R. A., Webb, D. F., Temmer, M., Odstrcil, D., Davies, J. A., Rollett, T., Luhmann, J. G., Nitta, N., Mulligan, T., Jensen, E. A., Forsyth, R., Lavraud, B., de Koning, C. A., Veronig, A. M., Galvin, A. B., Zhang, T. L., \& Anderson, B. J. 2012, Multi-point Shock and Flux Rope Analysis of Multiple Interplanetary Coronal Mass Ejections around 2010 August 1 in the Inner Heliosphere. Astrophys. J. 758, 10. http://dx.doi .org/10.1088/0004-637X/758/1/10DOI:10.1088/0004-637X/758/1/10.

Möstl, C., Amla, K., Hall, J. R., Liewer, P. C., De Jong, E. M., Colaninno, R. C., Veronig, A. M., Rollett, T., Temmer, M., Peinhart, V., Davies, J. A., Lugaz, N., Liu, Y. D., Farrugia, C. J., Luhmann, J. G., Vršnak, B., Harrison, R. A., \& Galvin, A. B. 2014, Connecting Speeds, Directions and Arrival Times of 22 Coronal Mass Ejections from the Sun to 1 AU. Astrophys. J. 787, 119. http://dx.doi.org/10.1088/0004-637X/787/2/119DOI:10.1088/0004$637 \mathrm{X} / 787 / 2 / 119$.

Muñoz-Jaramillo, A., Sheeley, N. R., Zhang, J., \& DeLuca, E. E. 2012, Calibrating 100 Years of Polar Faculae Measurements: Implications for the Evolution of the Heliospheric Magnetic Field. Astrophys. J. 753, 146. http://dx.doi.org/ 10.1088/0004-637X/753/2/146DOI:10.1088/0004-637X/753/2/146.

Muhr, N., Veronig, A. M., Kienreich, I. W., Vršnak, B., Temmer, M., \& Bein, B. M. 2014, Statistical Analysis of Large-Scale EUV Waves Observed by STEREO/EUVI. Solar Phys. 289, 4563. http://dx.doi.org/10.1007/s11207-014-0594-7DOI:10.1007/s11207-014-0594-7.

Mumford, S., Pérez-Suárez, D., Christe, S., Mayer, F., \& Hewett, R. J. 2013, Sunpy: Python for solar physicists. In: van der Walt, S., Millman, J., Huff, K. (eds.) Proceedings of the 12th Python in Science Conference, 74 .

Nakariakov, V. M., \& Verwichte, E. 2005, Coronal waves and oscillations. Living Rev. Solar Phys. 2(3). http://www.livingreviews.org/Irsp-2005-3.

Nelson, N. J., Brown, B. P., Brun, A. S., Miesch, M. S., \& Toomre, J. 2013, Magnetic Wreaths and Cycles in Convective Dynamos. Astrophys. J. 762, 73. http://dx.doi.org/ 10.1088/0004-637X/762/2/73DOI:10.1088/0004-637X/762/2/73.

Nelson, N. J., Brown, B. P., Brun, A., Miesch, M. S., \& Toomre, J. 2014, Buoyant Magnetic Loops Generated by Global Convective Dynamo Action. Solar Phys. 289, 441. http://dx.doi.org/10.1007/s11207-012-0221-4DOI:10.1007/s11207-012-0221-4.

Neuhäuser, R., \& Neuhäuser, D. L. 2015, Solar activity around AD 775 from aurorae and radiocarbon. Astronomische Nachrichten 336, 225. http://dx.doi.org/ 10.1002/asna.201412160DOI:10.1002/asna.201412160.

Nitta, N. V., Schrijver, C. J., Title, A. M., \& Liu, W. 2013, Large-scale Coronal Propagating Fronts in Solar Eruptions as Observed by the Atmospheric Imaging Assembly on Board the Solar Dynamics Observatory - an Ensemble Study. Astrophys. J. 776, 58.

Nogami, D., Notsu, Y., Honda, S., Maehara, H., Notsu, S., Shibayama, T., \& Shibata, K. 2014, Two sun-like superflare stars rotating as slow as the Sun*. PASJ 66, L4. http://dx.doi.org/10.1093/pasj/psu012DOI:10.1093/pasj/psu012.

Oberoi, D., Matthews, L. D., Cairns, I. H., Emrich, D., Lobzin, V., Lonsdale, C. J., Morgan, E. H., Prabu, T., Vedantham, H., Wayth, R. B., Williams, A., Williams, C., White, S. M., Allen, G., Arcus, W., Barnes, D., Benkevitch, L., Bernardi, G., Bowman, J. D., Briggs, F. H., Bunton, J. D., Burns, S., Cappallo, R. C., Clark, M. A., Corey, B. E., Dawson, M., DeBoer, D., De Gans, A., deSouza, L., Derome, M., Edgar, R. G., Elton, T., Goeke, R., Gopalakrishna, M. R., Greenhill, L. J., Hazelton, B., Herne, D., Hewitt, J. N., Kamini, 
P. A., Kaplan, D. L., Kasper, J. C., Kennedy, R., Kincaid, B. B., Kocz, J., Koeing, R., Kowald, E., Lynch, M. J., Madhavi, S., McWhirter, S. R., Mitchell, D. A., Morales, M. F., Ng, A., Ord, S. M., Pathikulangara, J., Rogers, A. E. E., Roshi, A., Salah, J. E., Sault, R. J., Schinckel, A., Udaya Shankar, N., Srivani, K. S., Stevens, J., Subrahmanyan, R., Thakkar, D., Tingay, S. J., Tuthill, J., Vaccarella, A., Waterson, M., Webster, R. L., \& Whitney, A. R. 2011, First Spectroscopic Imaging Observations of the Sun at Low Radio Frequencies with the Murchison Widefield Array Prototype. Astrophys. J., Lett. 728, L27. http://dx.doi.org/10.1088/2041-8205/728/2/L27DOI:10.1088/2041-8205/728/2/L27.

Ofman, L. 2010, Wave Modeling of the Solar Wind. Living Reviews in Solar Physics 7, 4. http://dx.doi.org/10.12942/lrsp-2010-4DOI:10.12942/lrsp-2010-4.

Oka, M., Krucker, S., Hudson, H. S., \& Saint-Hilaire, P. 2015, Electron Energy Partition in the Above-the-looptop Solar Hard X-Ray Sources. Astrophys. J. 799, 129. http://dx.doi.org/10.1088/0004-637X/799/2/129DOI:10.1088/0004-637X/799/2/129.

Olmedo, O., Vourlidas, A., Zhang, J., \& Cheng, X. 2012, Secondary Waves and/or the "Reflection" from and "Transmission" through a Coronal Hole of an Extreme Ultraviolet Wave Associated with the 2011 February 15 X2.2 Flare Observed with SDO/AIA and STEREO/EUVI. Astrophys. J. 756, 143. http://dx.doi.org/ 10.1088/0004-637X/756/2/143DOI:10.1088/0004-637X/756/2/143.

Otsuji, K., Sakurai, T., \& Kuzanyan, K. 2015, A statistical analysis of current helicity and twist in solar active regions over the phases of the solar cycle using the spectro-polarimeter data of Hinode. PASJ 67, 6. http://dx.doi.org/ 10.1093/pasj/psu130DOI:10.1093/pasj/psu130.

Panasenco, O., Martin, S. F., Velli, M., \& Vourlidas, A. 2013, Origins of Rolling, Twisting, and Non-radial Propagation of Eruptive Solar Events. Solar Phys. 287, 391. http://dx.doi.org/10.1007/s11207-012-0194-3DOI:10.1007/s11207-012-0194-3.

Passos, D., \& Charbonneau, P. 2014, Characteristics of magnetic solar-like cycles in a 3D MHD simulation of solar convection. $A \& A A$ 568, A113. http://dx.doi.org/10.1051/0004-6361/201423700DOI:10.1051/0004-6361/201423700.

Patsourakos, S., \& Vourlidas, A. 2012, On the Nature and Genesis of EUV Waves: A Synthesis of Observations from SOHO, STEREO, SDO, and Hinode (Invited Review). Solar Phys. $\mathbf{2 8 1}, 187$.

Patsourakos, S., Vourlidas, A., \& Stenborg, G. 2013, Direct Evidence for a Fast Coronal Mass Ejection Driven by the Prior Formation and Subsequent Destabilization of a Magnetic Flux Rope. Astrophys. J. 764, 125. http://dx.doi.org/ 10.1088/0004-637X/764/2/125DOI:10.1088/0004-637X/764/2/125.

Pavai, V. S., Arlt, R., Dasi-Espuig, M., Krivova, N., \& Solanki, S. 2015,. A $\& A$.

Pesnell, W. D., Thompson, B. J., \& Chamberlin, P. C. 2012, The Solar Dynamics Observatory (SDO). Solar Phys. 275, 3.

Petrie, G. J. D. 2012, The Abrupt Changes in the Photospheric Magnetic and Lorentz Force Vectors during Six Major Neutral-line Flares. Astrophys. J. 759, 50. http://dx.doi.org/10.1088/0004-637X/759/1/50DOI:10.1088/0004-637X/759/1/50.

Petrie, G. J. D. 2013, A Spatio-temporal Description of the Abrupt Changes in the Photospheric Magnetic and Lorentz-Force Vectors During the 15 February 2011 X2.2 Flare. Solar Phys. 287, 415. http://dx.doi.org/10.1007/s11207-012-0071-0DOI:10.1007/s11207012-0071-0.

Population reference bureau 2013, 2013 World population data sheet, PRB, Washington, DC.

Potgieter, M. 2013, Solar Modulation of Cosmic Rays. Living Reviews in Solar Physics 10, 3. http://dx.doi.org/10.12942/lrsp-2013-3; accessed 2015/09/02DOI:10.12942/lrsp2013-3; accessed 2015/09/02.

Raymond, J. C., McCauley, P. I., Cranmer, S. R., \& Downs, C. 2014, The Solar Corona as Probed by Comet Lovejoy (C/2011 W3). Astrophys. J. 788, 152. http://dx.doi.org/10.1088/0004-637X/788/2/152DOI:10.1088/0004-637X/788/2/152.

Reale, F., Orlando, S., Testa, P., Peres, G., Landi, E., \& Schrijver, C. J. 2013, Bright Hot Impacts by Erupted Fragments Falling Back on the Sun: A Template for Stellar Accretion. Science 341, 251. http://dx.doi.org/10.1126/science.1235692DOI:10.1126/science.1235692. 
Rempel, M. 2014, Numerical Simulations of Quiet Sun Magnetism: On the Contribution from a Small-scale Dynamo. Astrophys. J. $\mathbf{7 8 9}, 132$. http://dx.doi.org/10.1088/0004-637X/789/2/132DOI:10.1088/0004-637X/789/2/132.

Rempel, M. \& Cheung, M. C. M. 2014, Numerical Simulations of Active Region Scale Flux Emergence: From Spot Formation to Decay. Astrophys. J. 785, 90. http://dx.doi.org/10.1088/0004-637X/785/2/90DOI:10.1088/0004-637X/785/2/90.

Rollett, T., Möstl, C., Temmer, M., Veronig, A. M., Farrugia, C. J., \& Biernat, H. K. 2012, Constraining the Kinematics of Coronal Mass Ejections in the Inner Heliosphere with In-Situ Signatures. Solar Phys. 276, 293. http://dx.doi.org/10.1007/s11207-011-9897-0DOI:10.1007/s11207-011-9897-0.

Rubio da Costa, F., Kleint, L., Petrosian, V., Sainz Dalda, A., \& Liu, W. 2015, Solar Flare Chromospheric Line Emission: Comparison Between IBIS High-resolution Observations and Radiative Hydrodynamic Simulations. Astrophys. J. 804, 56. http://dx.doi.org/10.1088/0004-637X/804/1/56DOI:10.1088/0004-637X/804/1/56.

Russell, A. J. B., \& Fletcher, L. 2013, Propagation of Alfvénic Waves from Corona to Chromosphere and Consequences for Solar Flares. Astrophys. J. 765, 81. http://dx.doi.org/ 10.1088/0004-637X/765/2/81DOI:10.1088/0004-637X/765/2/81.

Schad, A., Timmer, J., \& Roth, M. 2013, Global Helioseismic Evidence for a Deeply Penetrating Solar Meridional Flow Consisting of Multiple Flow Cells. Astrophys. J., Lett. 778, L38. http://dx.doi.org/10.1088/2041-8205/778/2/L38DOI:10.1088/2041-8205/778/2/L38.

Scherrer, P. H., Schou, J., Bush, R. I., Kosovichev, A. G., Bogart, R. S., Hoeksema, J. T., Liu, Y., Duvall, T. L., Zhao, J., Title, A. M., Schrijver, C. J., Tarbell, T. D., \& Tomczyk, S. 2012, The Helioseismic and Magnetic Imager (HMI) Investigation for the Solar Dynamics Observatory (SDO). Solar Phys. 275, 207. http://dx.doi.org/10.1007/s11207-011-9834-2DOI:10.1007/s11207-011-9834-2.

Schmelz, J. T., Pathak, S., Brooks, D. H., Christian, G. M., \& Dhaliwal, R. S. 2014, Hot topic, warm loops, cooling plasma? multithermal analysis of active region loops. The Astrophysical Journal 795(2), 171. http://stacks.iop.org/0004-637X/795/i=2/a=171.

Schmidt, W., von der Lühe, O., Volkmer, R., Denker, C., Solanki, S. K., Balthasar, H., Bello Gonzalez, N., Berkefeld, T., Collados, M., Fischer, A., Halbgewachs, C., Heidecke, F., Hofmann, A., Kneer, F., Lagg, A., Nicklas, H., Popow, E., Puschmann, K. G., Schmidt, D., Sigwarth, M., Sobotka, M., Soltau, D., Staude, J., Strassmeier, K. G., \& Waldmann , T. A. 2012, The 1.5 meter solar telescope GREGOR. Astronomische Nachrichten 333, 796. http://dx.doi.org/10.1002/asna.201211725DOI:10.1002/asna.201211725.

Schmieder, B., Aulanier, G., \& Vršnak, B. 2015, Flare-CME Models: An Observational Perspective (Invited Review). Solar Phys. in press. http://dx.doi.org/ 10.1007/s11207-015-0712-1DOI:10.1007/s11207-015-0712-1.

Schrijver, C. J., \& Beer, J. 2014, Space Weather From Explosions on the Sun: How Bad Could It Be? EOS Transactions 95, 201. http://dx.doi.org/ 10.1002/2014E0240001DOI:10.1002/2014EO240001.

Sharykin, I. N., \& Kosovichev, A. G. 2014, Fine Structure of Flare Ribbons and Evolution of Electric Currents. Astrophys. J., Lett. 788, L18. http://dx.doi.org/ 10.1088/2041-8205/788/1/L18DOI:10.1088/2041-8205/788/1/L18.

Shen, Y., \& Liu, Y. 2012, Evidence for the Wave Nature of an Extreme Ultraviolet Wave Observed by the Atmospheric Imaging Assembly on Board the Solar Dynamics Observatory. Astrophys. J. 754, 7. http://dx.doi.org/10.1088/0004-637X/754/1/7DOI:10.1088/0004$637 \mathrm{X} / 754 / 1 / 7$.

Solanki, S. K., Krivova, N. A., \& Haigh, J. D. 2013, Solar Irradiance Variability and Climate. ARAESA 51, 311. http://dx.doi.org/ 10.1146/annurev-astro-082812-141007DOI:10.1146/annurev-astro-082812-141007.

Steinhilber, F., Abreu, J. A., Beer, J., Brunner, I., Christl, M., Fischer, H., Heikkila, U., Kubik, P. W., Mann, M., McCracken, K. G., Miller, H., Miyahara, H., Oerter, H., \& Wilhelms, F. 2012, 9,400 years of cosmic radiation and solar activity from ice cores and tree rings. Proceedings of the National Academy of Science 109, 5967. http://dx.doi.org/10.1073/pnas.1118965109DOI:10.1073/pnas.1118965109.

Su, J. T., Jing, J., Wang, S., Wiegelmann, T., \& Wang, H. M. 2014, Statistical Study of Free Magnetic Energy and Flare Productivity of Solar Active Regions. Astrophys. J. 788, 150. http://dx.doi.org/10.1088/0004-637X/788/2/150DOI:10.1088/0004-637X/788/2/150. 
Su, Y., Veronig, A. M., Holman, G. D., Dennis, B. R., Wang, T., Temmer, M., \& Gan, W. 2013, Imaging coronal magnetic-field reconnection in a solar flare. Nature Physics 9, 489. http://dx.doi.org/10.1038/nphys2675DOI:10.1038/nphys2675.

Sun, X., Hoeksema, J. T., Liu, Y., Wiegelmann, T., Hayashi, K., Chen, Q., \& Thalmann, J. 2012, Evolution of Magnetic Field and Energy in a Major Eruptive Active Region Based on SDO/HMI Observation. Astrophys. J. 748, 77. http://dx.doi.org/10.1088/0004-637X/748/2/77DOI:10.1088/0004-637X/748/2/77.

Sun, X., Bobra, M. G., Hoeksema, J. T., Liu, Y., Li, Y., Shen, C., Couvidat, S., Norton, A. A., \& Fisher, G. H. 2015, Why Is the Great Solar Active Region 12192 Flare-rich but CME-poor? Astrophys. J., Lett. 804, L28. http://dx.doi.org/ 10.1088/2041-8205/804/2/L28DOI:10.1088/2041-8205/804/2/L28.

Tadesse, T., Wiegelmann, T., \& MacNeice, P. J. 2015, Effect of the Size of the Computational Domain on Spherical Nonlinear Force-Free Modeling of a Coronal Magnetic Field Using SDO/HMI Data. Solar Phys. 290, 1159. http://dx.doi.org/ 10.1007/s11207-015-0664-5DOI:10.1007/s11207-015-0664-5.

Tan, B. 2014, Coronal Heating Driven by a Magnetic Gradient Pumping Mechanism in Solar Plasmas. Astrophys. J. 795, 140. http://dx.doi.org/ 10.1088/0004-637X/795/2/140DOI:10.1088/0004-637X/795/2/140.

Temmer, M., Vršnak, B., Rollett, T., Bein, B., de Koning, C. A., Liu, Y., Bosman, E., Davies, J. A., Möstl, C., Žic, T., Veronig, A. M., Bothmer, V., Harrison, R., Nitta, N., Bisi, M., Flor, O., Eastwood, J., Odstrcil, D., \& Forsyth, R. 2012, Characteristics of Kinematics of a Coronal Mass Ejection during the 2010 August 1 CME-CME Interaction Event. Astrophys. J. 749, 57. http://dx.doi.org/10.1088/0004-637X/749/1/57DOI:10.1088/0004$637 \mathrm{X} / 749 / 1 / 57$.

Temmer, M., Veronig, A. M., Peinhart, V., \& Vršnak, B. 2014, Asymmetry in the CMECME Interaction Process for the Events from 2011 February 14-15. Astrophys. J. 785, 85. http://dx.doi.org/10.1088/0004-637X/785/2/85DOI:10.1088/0004-637X/785/2/85.

Testa, P., De Pontieu, B., Allred, J., Carlsson, M., Reale, F., Daw, A., Hansteen, V., Martinez-Sykora, J., Liu, W., DeLuca, E. E., Golub, L., McKillop, S., Reeves, K., Saar, S., Tian, H., Lemen, J., Title, A., Boerner, P., Hurlburt, N., Tarbell, T. D., Wuelser, J. P., Kleint, L., Kankelborg, C., \& Jaeggli, S. 2014, Evidence of nonthermal particles in coronal loops heated impulsively by nanoflares. Science 346(6207). http://dx.doi.org/10.1126/science.1255724DOI:10.1126/science.1255724. http://www.sciencemag.org/content/346/6207/1255724.abstract.

Thalmann, J. K., Su, Y., Temmer, M., \& Veronig, A. M. 2015, The Confined X-class Flares of Solar Active Region 2192. Astrophys. J., Lett. 801, L23. http://dx.doi.org/10.1088/2041-8205/801/2/L23DOI:10.1088/2041-8205/801/2/L23.

Tian, H., Li, G., Reeves, K. K., Raymond, J. C., Guo, F., Liu, W., Chen, B., \& Murphy, N. A. 2014, Imaging and Spectroscopic Observations of Magnetic Reconnection and Chromospheric Evaporation in a Solar Flare. Astrophys. J., Lett. 797, L14. http://dx.doi .org/10.1088/2041-8205/797/2/L14DOI:10.1088/2041-8205/797/2/L14.

Tiwari, S. K., Falconer, D. A., Moore, R. L., Venkatakrishnan, P., Winebarger, A. R., \& Khazanov, I. G. 2015, Near-Sun speed of CMEs and the magnetic nonpotentiality of their source active regions. Geophys. Res. Lett. 42, 5702. http://dx.doi.org/10.1002/2015GL064865DOI:10.1002/2015GL064865.

Tomczyk, S., \& McIntosh, S. W. 2009, Time-Distance Seismology of the Solar Corona with CoMP. Astrophys. J. 697, 1384. http://dx.doi.org/ 10.1088/0004-637X/697/2/1384DOI:10.1088/0004-637X/697/2/1384.

Tomczyk, S., McIntosh, S. W., Keil, S. L., Judge, P. G., Schad, T., Seeley, D. H., \& Edmondson, J. 2007, Alfvén Waves in the Solar Corona. Science 317, 1192. http://dx.doi.org/10.1126/science.1143304DOI:10.1126/science.1143304.

Tomczyk, S., Card, G. L., Darnell, T., Elmore, D. F., Lull, R., Nelson, P. G., Streander, K. V., Burkepile, J., Casini, R., \& Judge, P. G. 2008, An Instrument to Measure Coronal Emission Line Polarization. Solar Phys. 247, 411. http://dx.doi.org/10.1007/s11207-007-9103-6DOI:10.1007/s11207-007-9103-6.

Török, T., Temmer, M., Valori, G., Veronig, A. M., van Driel-Gesztelyi, L., \& Vršnak, B. 2013, Initiation of Coronal Mass Ejections by Sunspot Rotation. Solar Phys. 286, 453. http://dx.doi.org/10.1007/s11207-013-0269-9DOI:10.1007/s11207-013-0269-9. 
Upton, L., \& Hathaway, D. H. 2014, Effects of Meridional Flow Variations on Solar Cycles 23 and 24. Astrophys. J. 792, 142. http://dx.doi.org/ 10.1088/0004-637X/792/2/142DOI:10.1088/0004-637X/792/2/142.

Usoskin, I. G. 2008, A History of Solar Activity over Millennia. Living Reviews in Solar Physics $\mathbf{5}, 3$.

Usoskin, I. G., Kromer, B., Ludlow, F., Beer, J., Friedrich, M., Kovaltsov, G. A., Solanki, S. K., \& Wacker, L. 2013, The AD775 cosmic event revisited: the Sun is to blame. A 6 A 552, L3. http://dx.doi.org/10.1051/0004-6361/201321080DOI:10.1051/0004-6361/201321080.

van Ballegooijen, A. A., Asgari-Targhi, M., \& Berger, M. A. 2014, On the Relationship Between Photospheric Footpoint Motions and Coronal Heating in Solar Active Regions. Astrophys. J. 787, 87. http://dx.doi.org/10.1088/0004-637X/787/1/87DOI:10.1088/0004$637 \mathrm{X} / 787 / 1 / 87$.

van Ballegooijen, A. A., Asgari-Targhi, M., Cranmer, S. R., \& DeLuca, E. E. 2011, Heating of the Solar Chromosphere and Corona by Alfvén Wave Turbulence. Astrophys. J. 736, 3. http://dx.doi .org/10.1088/0004-637X/736/1/3DOI:10.1088/0004-637X/736/1/3.

Van Doorsselaere, T., Nakariakov, V. M., \& Verwichte, E. 2008, Detection of Waves in the Solar Corona: Kink or Alfvén? Astrophys. J., Lett. 676, L73. http://dx.doi .org/10.1086/587029DOI:10.1086/587029.

van Noort, M. J. \& Rouppe van der Voort, L. H. M. 2008, Stokes imaging polarimetry using image restoration at the Swedish 1-m solar telescope. A\&B $4 \mathbf{4 8 9}, 429$. http://dx.doi.org/10.1051/0004-6361:200809959DOI:10.1051/0004-6361:200809959.

Varady, M., Karlický, M., Moravec, Z., \& Kašparová, J. 2014, Modifications of thicktarget model: re-acceleration of electron beams by static and stochastic electric fields. $A \& \mathcal{E} A$ 563, A51. http://dx.doi.org/10.1051/0004-6361/201322391DOI:10.1051/0004$6361 / 201322391$.

Vemareddy, P., Ambastha, A., \& Maurya, R. A. 2012, On the Role of Rotating Sunspots in the Activity of Solar Active Region NOAA 11158. Astrophys. J. 761, 60. http://dx.doi.org/10.1088/0004-637X/761/1/60DOI:10.1088/0004-637X/761/1/60.

Verwichte, E., Van Doorsselaere, T., Foullon, C., \& White, R. S. 2013, Coronal Alfvén Speed Determination: Consistency between Seismology Using AIA/SDO Transverse Loop Oscillations and Magnetic Extrapolation. Astrophys. J. 767, 16. http://dx.doi.org/10.1088/0004-637X/767/1/16DOI:10.1088/0004-637X/767/1/16.

Vourlidas, A., Lynch, B. J., Howard, R. A., \& Li, Y. 2013, How Many CMEs Have Flux Ropes? Deciphering the Signatures of Shocks, Flux Ropes, and Prominences in Coronagraph Observations of CMEs. Solar Phys. 284, 179. http://dx.doi.org/10.1007/s11207-012-0084-8DOI:10.1007/s11207-012-0084-8.

Vršnak, B., Žic, T., Vrbanec, D., Temmer, M., Rollett, T., Möstl, C., Veronig, A., Čalogović, J., Dumbović, M., Lulić, S., Moon, Y.-J., \& Shanmugaraju, A. 2013, Propagation of Interplanetary Coronal Mass Ejections: The Drag-Based Model. Solar Phys. 285, 295. http://dx.doi.org/10.1007/s11207-012-0035-4DOI:10.1007/s11207-012-0035-4.

Vršnak, B., Temmer, M., Žic, T., Taktakishvili, A., Dumbović, M., Möstl, C., Veronig, A. M., Mays, M. L., \& Odstrčil, D. 2014, Heliospheric Propagation of Coronal Mass Ejections: Comparison of Numerical WSA-ENLIL+Cone Model and Analytical Drag-based Model. 213, 21. http://dx.doi.org/10.1088/0067-0049/213/2/21DOI:10.1088/00670049/213/2/21.

Wang, S., Liu, C., \& Wang, H. 2012, The Relationship between the Sudden Change of the Lorentz Force and the Magnitude of Associated Flares. Astrophys. J., Lett. 757, L5. http://dx.doi.org/10.1088/2041-8205/757/1/L5DOI:10.1088/2041-8205/757/1/L5.

Wang, S., Liu, C., Liu, R., Deng, N., Liu, Y., \& Wang, H. 2012, Response of the Photospheric Magnetic Field to the X2.2 Flare on 2011 February 15. Astrophys. J., Lett. 745, L17. http://dx.doi.org/10.1088/2041-8205/745/2/L17DOI:10.1088/2041-8205/745/2/L17.

Wang, W., Yan, Y., Liu, D., Chen, Z., Su, C., Liu, F., Geng, L., Chen, L., \& Du, J. 2013, Calibration and Data Processing for a Chinese Spectral Radioheliograph in the Decimeter Wave Range. PASJ 65, 18. http://dx.doi.org/ 10.1093/pasj/65.sp1.S18DOI:10.1093/pasj/65.sp1.S18. 
Warren, H. P. 2014, Measurements of Absolute Abundances in Solar Flares. Astrophys. J., Lett. 786, L2. http://dx.doi.org/10.1088/2041-8205/786/1/L2DOI:10.1088/2041$8205 / 786 / 1 / \mathrm{L} 2$.

Warren, H. P., Winebarger, A. R., \& Brooks, D. H. 2012, A systematic survey of hightemperature emission in solar active regions. The Astrophysical Journal 759(2), 141. ISBN 0004-637X. http://stacks.iop.org/0004-637X/759/i=2/a=141.

Watanabe, K., Shimizu, T., Masuda, S., Ichimoto, K., \& Ohno, M. 2013, Emission Height and Temperature Distribution of White-light Emission Observed by Hinode/SOT from the 2012 January 27 X-class Solar Flare. Astrophys. J. 776, 123. http://dx.doi.org/10.1088/0004-637X/776/2/123DOI:10.1088/0004-637X/776/2/123.

Webb, D. F., \& Howard, T. A. 2012, Coronal Mass Ejections: Observations. Living Reviews in Solar Physics 9, 3. http://dx.doi.org/10.12942/lrsp-2012-3DOI:10.12942/lrsp-2012-3.

Wedemeyer-Böhm, S., Scullion, E., Steiner, O., Rouppe van der Voort, L., de La Cruz Rodriguez, J., Fedun, V., \& Erdélyi, R. 2012, Magnetic tornadoes as energy channels into the solar corona. Nature 486, 505. http://dx.doi.org/ 10.1038/nature11202DOI:10.1038/nature11202.

Welsch, B. T. 2015, The photospheric poynting flux and coronal heating. Publications of the Astronomical Society of Japan 67(2). http://dx.doi.org/ 10.1093/pasj/psu151DOI:10.1093/pasj/psu151. http://pasj.oxfordjournals.org/content/67/ 2/18.abstract.

White, R. S., \& Verwichte, E. 2012, Transverse coronal loop oscillations seen in unprecedented detail by AIA/SDO. A\&SA 537, A49. http://dx.doi.org/ 10.1051/0004-6361/201118093DOI:10.1051/0004-6361/201118093.

Wiegelmann, T., Thalmann, J. K., \& Solanki, S. K. 2014, The magnetic field in the solar atmosphere. Astron. and Astrophys. Rev. 22, 78. http://dx.doi.org/ 10.1007/s00159-014-0078-7DOI:10.1007/s00159-014-0078-7.

Winebarger, A. R., Cirtain, J., Golub, L., DeLuca, E., Savage, S., Alexander, C., \& Schuler, T. 2014, Discovery of Finely Structured Dynamic Solar Corona Observed in the Hi-C Telescope. Astrophys. J., Lett. 787, L10. http://dx.doi.org/ 10.1088/2041-8205/787/1/L10DOI:10.1088/2041-8205/787/1/L10.

Withbroe, G. L., \& Noyes, R. W. 1977, Mass and energy flow in the solar chromosphere and corona. ARA\&A 15, 363. http://dx.doi.org/ 10.1146/annurev .aa.15.090177.002051DOI:10.1146/annurev.aa.15.090177.002051.

Woods, T. N., Eparvier, F. G., Hock, R., Jones, A. R., Woodraska, D., Judge, D., Didkovsky, L., Lean, J., Mariska, J., Warren, H., McMullin, D., Chamberlin, P., Berthiaume, G., Bailey, S., Fuller-Rowell, T., Sojka, J., Tobiska, W. K., \& Viereck, R. 2012, Extreme Ultraviolet Variability Experiment (EVE) on the Solar Dynamics Observatory (SDO): Overview of Science Objectives, Instrument Design, Data Products, and Model Developments. Solar Phys. 275, 115. http://dx.doi .org/10.1007/s11207-009-9487-6DOI:10.1007/s11207-009-9487-6.

Yan, Y., Zhang, J., Wang, W., Liu, F., Chen, Z., \& Ji, G. 2009, The Chinese Spectral Radioheliograph - CSRH. Earth Moon and Planets 104, 97. http://dx.doi.org/ 10.1007/s11038-008-9254-yDOI:10.1007/s11038-008-9254-y.

Yeates, A. R. 2014, Coronal Magnetic Field Evolution from 1996 to 2012: Continuous Non-potential Simulations. Solar Phys. 289, 631. http://dx.doi.org/ 10.1007/s11207-013-0301-0DOI:10.1007/s11207-013-0301-0.

Young, P. R., Doschek, G. A., Warren, H. P., \& Hara, H. 2013, Properties of a Solar Flare Kernel Observed by Hinode and SDO. Astrophys. J. 766, 127. http://dx.doi .org/10.1088/0004-637X/766/2/127DOI:10.1088/0004-637X/766/2/127.

Zhang, J., \& Liu, Y. 2011, Ubiquitous Rotating Network Magnetic Fields and Extreme-ultraviolet Cyclones in the Quiet Sun. Astrophys. J., Lett. 741, L7. http://dx.doi .org/10.1088/2041-8205/741/1/L7DOI:10.1088/2041-8205/741/1/L7.

Zhao, J., Bogart, R. S., Kosovichev, A. G., Duvall, T. L. Jr., \& Hartlep, T. 2013, Detection of Equatorward Meridional Flow and Evidence of Double-cell Meridional Circulation inside the Sun. Astrophys. J., Lett. 774, L29. http://dx.doi.org/ 10.1088/2041-8205/774/2/L29DOI:10.1088/2041-8205/774/2/L29. 
Zharkov, S., Green, L. M., Matthews, S. A., \& Zharkova, V. V. 2013, Properties of the 15 February 2011 Flare Seismic Sources. Solar Phys. 284, 315. http://dx.doi.org/ 10.1007/s11207-012-0169-4DOI:10.1007/s11207-012-0169-4.

Zuccarello, F. P., Bemporad, A., Jacobs, C., Mierla, M., Poedts, S., \& Zuccarello, F. 2012, The Role of Streamers in the Deflection of Coronal Mass Ejections: Comparison between STEREO Three-dimensional Reconstructions and Numerical Simulations. Astrophys. J. 744, 66. http://dx.doi.org/10.1088/0004-637X/744/1/66DOI:10.1088/0004$637 \mathrm{X} / 744 / 1 / 66$. 